Portland State University

PDXScholar

Summer 1-1-2012

\title{
Effects of Reactive Oxygen Species on Life History Traits of Caenorhabditis elegans
}

Samson William Smith

Portland State University

Follow this and additional works at: https://pdxscholar.library.pdx.edu/open_access_etds

Part of the Biology Commons, Other Genetics and Genomics Commons, and the Plant Breeding and Genetics Commons

Let us know how access to this document benefits you.

\section{Recommended Citation}

Smith, Samson William, "Effects of Reactive Oxygen Species on Life History Traits of Caenorhabditis elegans" (2012). Dissertations and Theses. Paper 712.

https://doi.org/10.15760/etd.712

This Thesis is brought to you for free and open access. It has been accepted for inclusion in Dissertations and Theses by an authorized administrator of PDXScholar. Please contact us if we can make this document more accessible: pdxscholar@pdx.edu. 
Effects of Reactive Oxygen Species on Life History Traits of Caenorhabditis elegans

by

Samson William Smith

A thesis submitted in partial fulfillment of the requirements for the degree of

Master of Science

in

Biology

Thesis Committee

Suzanne Estes, Chair

Michael Bartlett

Bradley Buckley

Portland State University

(C)2012 


\begin{abstract}
Evolutionary life history theory predicts that tradeoffs among fitness-related phenotypes will occur as a result of resource limitations and/or physiological constraints. Such tradeoffs are defined as the $\operatorname{cost}(\mathrm{s})$ incurred on one component of fitness (e.g., reproduction) by the increased expression of another fitness-related trait (e.g., longevity). Only recently have researchers begun to investigate the mechanistic bases of life history tradeoffs. A recent proposal is that reactive oxygen species (ROS) have a central role in shaping life history traits and tradeoffs. Research on disparate animal taxa has highlighted strong correlations between oxidative stress resistance and fitness-related life history traits, for example. Here, I use the model organism Caenorhabditis elegans to test several hypotheses concerning the effects of ROS on life history traits and the manifestation of life history tradeoffs. Additionally, I use heat stress and an alternate food source to explore the responses of life history traits to other forms of physiological stress. Relative fitness and other traits related to reproduction were found to be affected in mostly negative ways by increasing oxidative insult. Lifespan was surprisingly unaffected by oxidative stress, but was modified by temperature. In vivo ROS levels as measured by fluorescent microscopy reveal a tradeoff between antioxidant production and reproduction in this species.
\end{abstract}




\section{Acknowledgements}

A big thank you goes out to all my friends and family for helping me succeed in ways I never imagined. I am also extremely grateful for the assistance I received from many undergraduate researchers. Kiley, our friendship has gotten stronger since we bonded over music and short-tempered, suicidal cockroaches, but has remained just as weird and fun ever since; thanks for everything. Finally, this thesis would not have been possible without the unique knowledge, support, and mentoring font that is Suzanne Estes.

"Life ain't nothin' but a funny, funny riddle."

- John Martin Sommers 


\section{Table of Contents}

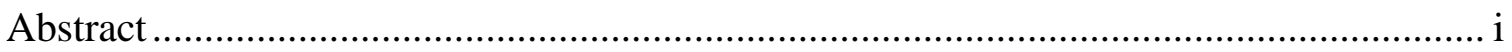

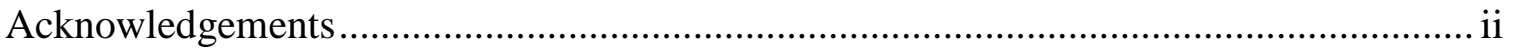

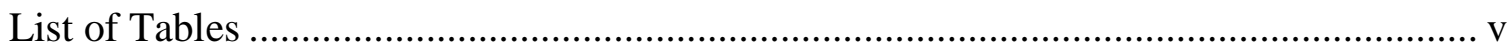

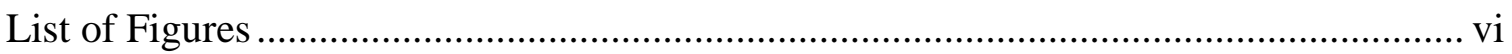

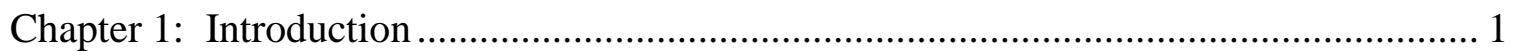

Chapter 2: Effects of Reactive Oxygen Species on Life History Traits of Caenorhabditis

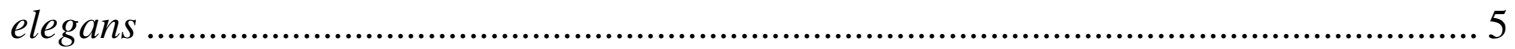

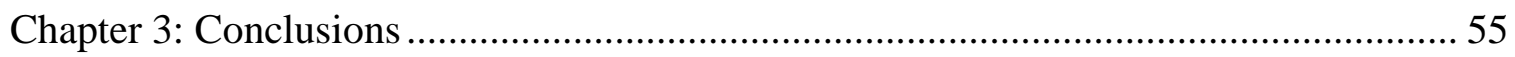

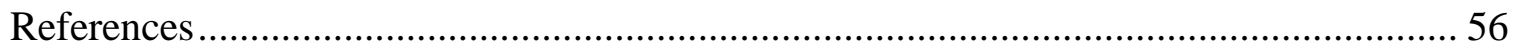


List of Tables

Table 1. Life History Traits at 25

${ }^{\circ} \mathrm{C}$

40

Table 2. Life History Traits at 20

${ }^{\circ} \mathrm{C}$

41

Table 3. Results of Mixed Model for Source of Variation in Time to First Reproduction

Assays

42

Table 4. Spearman Pairwise Correlations ( $\rho$ ) and Individual Trait Variances at 25 ${ }^{\circ} \mathrm{C}$

42

Table 5. Spearman Pairwise Correlations ( $\rho$ ) and Individual Trait Variances at 20 ${ }^{\circ} \mathrm{C}$

43 


\section{List of Figures}

Figure 1. Total reproductive output in C. elegans is modified by temperature, oxidative stress, and

diet.

44

Figure 2. Reproductive schedules for each treatment level recorded as the mean number of surviving offspring per day of reproduction.

45

Figure 3. Time to reproduction determined by the hour of the first egg laid at control (squares) and three oxidative stress treatment levels (low $=$ circles, medium $=$ triangles, high $=\mathrm{x}$ ) for worms raised under two temperature regimes and fed live $E$.

coli.

46

Figure 4. Mean lifespan as measured by total number of days lived beyond L1 stage arrest for worms experiencing (A) $25{ }^{\circ} \mathrm{C}$ and fed live OP50-1 E. coli. ANOVA testing indicated no significant variation among treatment means $(\mathrm{F}=0.49, \mathrm{p}=0.69)(\mathrm{B}) 20^{\circ} \mathrm{C}$ and fed UV-killed $E$.

coli.

47

Figure 5. Survival curves for control worms raised at $25^{\circ} \mathrm{C}$ on live E. coli (solid) or on UV-killed E. coli at $20^{\circ} \mathrm{C}$ (dashed).

48

Figure 6. Relative fitness ( $\omega$ ) of worms experiencing (A) $25^{\circ} \mathrm{C}$ and fed live E. coli and (B) $20{ }^{\circ} \mathrm{C}$ and fed UV-killed $E$.

coli.

49

Figure 7. Spearman pairwise rank correlations for life history traits at each oxidative stress treatment level for worms grown at $25{ }^{\circ} \mathrm{C}$ and fed live OP50$1 E$.

coli.

50

Figure 8. Spearman pairwise rank correlations for life history traits at each oxidative stress treatment level for worms grown at $25{ }^{\circ} \mathrm{C}$ and fed live OP50$1 E$. 
coli.

51

Figure 9. Spearman pairwise rank correlations for life history traits at each oxidative stress treatment level for worms grown at $20^{\circ} \mathrm{C}$ and fed UV-killed OP50-1 E.

coli.

52

Figure 10. Spearman pairwise rank correlations for life history traits at each treatment level for worms grown at $20^{\circ} \mathrm{C}$ and consuming UV-killed OP501.

53

Figure 11. Reactive oxygen species (ROS) level measured as average maximum pixel intensity

(RFU). 


\section{Chapter 1: Introduction}

An organism that is capable of unlimited reproduction over the course of an infinitely long lifespan exists neither in nature nor in the laboratory. Although this might seem a trivial observation, the absence of such a "Darwinian demon" (c.f., Reznick et al., 2000) suggests that there is a constraint on fitness-related traits preventing their boundless evolution. Constraints on fitness come from both extrinsic and intrinsic forces (2). Extrinsic selective pressures on life history traits originate from environmental sources including predation and food acquisition. For example, strong predation may result in faster maturation time and smaller size at reproduction (3). By contrast, intrinsic sources of selection on life history traits are often associated with the concept of life history tradeoffs (4). Life history tradeoffs exist when increases in one component of fitness result directly or indirectly in the decline of another component of fitness. The negative correlations between such fitness components will prevent the successful maximization of both traits simultaneously; the effect of these negative genetic correlations is that both affected traits are constrained to a local maximum within an phenotypic adaptive landscape $(5,6)$.

The proximal mechanisms or constraints that determine the existence and magnitude of life history tradeoffs (i.e., intrinsic sources) are not well understood. Pearl

(7) proposed that some finite, or 'vital', resource was being depleted over the lifespan of an organism that, when fully expended, led to death. This hypothesis makes intuitive sense with respect to specific metabolic rate and maximum lifespan as there has been shown to be a strong negative correlation between these two traits in a wide range of 
mammalian taxa (8). The idea that some aspect of metabolism negatively impacts life history traits, primarily senescence, is now incorporated into the oxidative stress theory of aging. This theory expands upon the mitochondrial biological clock/free radical theory of aging put forth by Harman (9) and states that the mere act of energy production causes senescent decline of organisms through the inevitable production of reactive oxygen species (ROS) (10). ROS are known to cause DNA lesions, lipid peroxidation and other forms of damage that are expected to reduce fitness in age-related manner (11). The production of ROS may thus present a cost in higher energy production (directed toward antioxidant defense) and has recently been hypothesized to play a key role in life history tradeoffs (12). In support of this idea, metabolic rate is also known to correlate well with antioxidant protein expression (13). Life history tradeoffs are well discussed in theoretical literature although these tradeoffs are not always revealed in laboratory experiments (1). Stearns (1989) identified two mechanisms that would generate nonnegative genetic correlations, both of which rely on internal environmental conditions such as genotype specific growth rate and resource allocation. In short: negative correlations might only be revealed in poor or stressful conditions.

Caenorhabditis elegans is a small free-living soil nematode that was first isolated in decaying plant material in Bristol, UK, and has since been repeatedly isolated mainly from compost sites near human populations. This small (about $1.5 \mathrm{~mm}$ in length) transparent and primarily hermaphroditic nematode quickly became a widely used model organism after one enterprising researcher, Sydney Brenner, suggested that it was ideal for studies linking genetics to neurology and behavior (14). Since then, C. elegans has 
served as a model for studies ranging from developmental genetics to environmental biomonitoring (15) and aging (16), and has been fully developed as a genetic model organism. Male homosexuality has also been documented in a wild isolate making it perhaps one of the only small invertebrates to have been observed engaging in this behavior (17) and illustrates the complex behavioral and genetic similarities it may share with more physiologically and genetically complex animals. The short life cycle of $C$. elegans also contributes to its value as a general research model and makes this species an ideal model for investigating outstanding questions in evolutionary life history theory.

Here, I examined the role that ROS might play in mediating life history tradeoffs in C. elegans by subjecting individual hermaphrodites to increasing levels of chronic oxidative stress caused by paraquat exposure (Chapter 2). I also performed additional assays using a combination of oxidative stress and mild heat stress in a further attempt to reveal negative correlations among life history traits. I found few significant negative fitness-related trait correlations at either temperature treatment as oxidative stress increased and thus found little support for the hypothesis that exogenous ROS mediates life history tradeoffs. I further interpret these findings within the context of data on in vivo ROS levels. Both oxidative and heat stress did produce substantial effects on certain individual life history traits. For example, the most surprising finding of this study was that although temperature had a significant effect on average nematode lifespan, the level of oxidative stress had no effect on this trait. Furthermore, I showed that worms fed UVkilled bacteria had severely reduced fitness as oxidative stress increased compared to worms fed living E. coli. These results suggest that $C$. elegans may receive significant 
antioxidant supplementation from living bacteria, or that living bacteria initiate an innate antioxidant response in these worms, either of which results in increased fitness when experiencing chronic oxidative stress. Finally, I summarize the major findings of this thesis and discuss its implications as well as work in progress and directions for future research (Chapter 3). 


\section{Chapter 2: Effects of Reactive Oxygen Species on Life History Traits of Caenorhabditis elegans}

\section{Introduction}

Evolutionary life history theory predicts that tradeoffs among fitness-related phenotypes will occur as a result of resource limitations and/or physiological constraints $(4,12,18,19)$. Life history tradeoffs are typically defined as the cost(s) incurred on one component of fitness either concurrently or subsequently by the increased expression of another trait in a kind of zero-sum game. Without such tradeoffs we should expect the evolution of a "Darwinian demon" (c.f, Reznick et al., 2000), or an organism that could reproduce throughout its long lifespan and avoid age-correlated senescent decline. Commonly demonstrated tradeoffs include the so-called 'costs of reproduction' whereby lifetime fecundity is negatively correlated to other traits such as somatic maintenance (18). In this example, individuals who produce more offspring should have predictably shorter lifespans, accelerated aging, and/or be more susceptible to stress than individuals who have fewer offspring $(20,21)$. In contrast, researchers have also reported positive or variable correlations between traits where classic theory would predict the existence of strong negative correlations $(1,22)$. There has been much debate as to why the negative correlations predicted by theory are not clearly obtained in empirical studies (e.g., 24). With specific regard to laboratory studies of tradeoffs, positive correlations among life history traits may be obtained when resource budgets are expanded as is the case under ideal laboratory conditions with unlimited resources and optimal environmental conditions; however, reducing these resource budgets might illuminate true tradeoffs $(1,24)$. Accordingly, negative correlations among life history traits have been revealed 
experimentally by manipulating resource intake, brood size, or modulation of other stressors while tracking correlations among life history traits $(18,21,25,26)$.

The currency or constraining resource that mediates life history tradeoffs has been hypothesized to be energetic, hormonal, and/or the result of more direct negative effects on one trait from the expression of another trait (see reference 4 for a detailed review on proposed physiological causes of life history tradeoffs). A growing body of literature suggests that reactive oxygen species (ROS) may play a central role in many aspects of life history evolution (27), including tradeoffs and aging. Indeed, ROS have been proposed to directly mediate life history tradeoffs by initiating oxidative stress, initiating macromolecular damage that would presumably require the allocation of resources away from reproduction and to somatic maintenance $(12,27)$. Oxidative stress may be defined as the oxidative damage to macromolecular cellular components resulting from an inequity between the production of ROS and the production and/or procurement of protective antioxidants (1). The role of general oxidative stress in age-correlated cellular damage was initially highlighted by Harman (1956) whose hypothesis was an extension of Pearl's (1928) rate of living theory. Harman's proposal implicated mitochondria, the primary source of endogenous ROS, as a major cause of senescence through the production of free radicals by the electron transport chain (ETC). This hypothesis was later incorporated into a more general oxidative stress theory of aging $(11,29)$. ROS are a natural byproduct of aerobic energy (ATP) production that occurs via the mitochondrial ETC (30). Endogenous ROS production from mitochondrial respiration has been empirically shown to result in the buildup of age-correlated nucleic acid, lipid, 
carbohydrate and protein damage (31-36). For example (32) demonstrated high steady state levels of oxidative damage to both mitochondrial and nuclear DNA as indicated by the detection of the oxidatively modified guanine base-derived 8-oxo-dG in rat liver. In a model where ROS mediate life history tradeoffs, an organism must satisfy the requirements of its dependence on ATP production to fuel cellular functions and also be capable of regulating the levels of detrimental ROS byproducts thereby creating a possible tradeoff between these somatic maintenance (systems inhibiting the agecorrelated decline of somatic tissues), growth and reproduction.

Research on ETC dysfunctional mutants has shed light on the link between mitochondrial function (e.g., ROS generation) and life histories (37-42). For example, mutations affecting protein subunits of the ETC have been shown to have significant, mostly detrimental, effects on various components of fitness, including development, lifespan, fecundity and responses to stressors such as heat, oxidative stress, and UV irradiation (43). These effects are thought to result from increased mitochondrial ROS production. In studies of the nematode, Caenorhabditis elegans, short-lived ETC mutants have shown increased sensitivity to redox cycling compounds. Conversely, a trend between increased resistance to pro-oxidants and increased longevity has been established $(42,43)$; for example a long-lived C. elegans ETC mutant, isp-1, is resistant to paraquat, a ROS-generating herbicide (44). Many other studies have highlighted the link between fitness-related traits and either ROS production or oxidative susceptibility and provide support for the idea that oxidative stress plays a central role in lifespan determination and that it may be at the heart of life history tradeoffs $(11,12,27,41)$. 
However, ROS and other free radicals are also known to have beneficial and vital intra- and inter-cellular roles in the immune system and as second messengers in signal transduction cascades $(36,45,46)$. Studies that have modified antioxidant levels have produced counterintuitive results with respect to lifespan (47-52), but generally support the idea that antioxidants (both exogenous and endogenous) protect against high and acute oxidative stress $(49,53)$. Taken together, these findings suggest a complex relationship between balancing fitness by minimizing oxidative damage and maintaining the beneficial roles associated with ROS signaling.

Here, I investigated the link between oxidative stress and life history tradeoffs by measuring life history characters in Caenorhabditis elegans nematodes exposed to two stressors, oxidative stress and thermal stress. For the oxidative stress treatments, I exposed nematodes to paraquat, alternatively known as methyl viologen - an herbicide that generates superoxide anions in vivo $(54,55)$ and is commonly used experimentally to test oxidative stress resistance (56-58). Next, I examined whether and how life history traits would respond to a combination of oxidative and thermal stress. Increased temperatures (relative to the lab-determined optimum of $20^{\circ} \mathrm{C}$ ) are known to affect development, fecundity, and lifespan in C. elegans (22). I subjected worms to $25^{\circ} \mathrm{C}$, a temperature at which wild-type nematodes are known to have accelerated development resulting in a shorter period of egg-laying and a significantly shorter lifespan (22). Unfortunately, the results of these experiments are confounded by differences in nematode diet, an issue that is being addressed as part of ongoing research (see Chapter 3). 
Additionally, I conducted in vivo measures of ROS levels for individual nematodes exposed to each oxidative stress and temperature regime to understand the effect of these stressors on cellular ROS levels. These experiments were conducted using nematodes experiencing identical dietary regimes. This portion of my study was motivated by the work of Tawe et al. (1998) and (59) showing the effect of age on physiological responses to stress. Tawe et al., 1998, subjected C. elegans to short periods of high concentrations of paraquat and found an increased expression of antioxidant proteins in larval stage worms relative to untreated control worms. Interestingly, they found no difference in antioxidant levels in mixed-age cohorts that were exposed to the same level of oxidative insult; i.e., older worms exhibited a dampened transcriptional response to acute oxidative stress compared to larval worms. Based on these findings, I hypothesized that net in vivo ROS levels should decline with increasing paraquat treatment (as a result of the upregulation of antioxidant mechanisms), at least until treatment levels beyond which nematodes could no longer balance both reproduction and somatic maintenance. Additionally, I expected that ROS levels within each stress treatment would be lower in larval worms compared to adult worms due to the increased antioxidant production of the former. In summary, these experiments were expected to reveal life history tradeoffs by reducing the animals' total resource budget and also to indicate how animals prioritize their resources under increasing levels of physiological stress.

In stark contrast to my expectations, I found that increasing levels of oxidative or thermal stress failed to reveal the life history tradeoffs predicted by theory. In fact, 
correlations between phenotypic traits (e.g., lifespan vs. late reproduction) became strongly positive under the most stressful treatment for the fitness-related traits that I measured. Both forms of stress did, however, negatively impact individual reproductive fitness traits as stress levels increased. On the other hand, average nematode lifespan was surprisingly resilient to the forms of stress applied in this study. In agreement with my predictions, in vivo ROS levels were indeed lower in larval worms relative to adults from the same treatment cohort and in larval worms experiencing the highest oxidative stress treatment. This result indicates that strong antioxidant systems, which may have been supplemented through dietary means, are capable of staving off the accumulated macromolecular damage associated with senescence and that chronic oxidative stress impacts reproduction more than lifespan in this system as illustrated by resultant life history assay data. This final observation suggests that a life history tradeoff mediated by ROS occurs between reproductive fitness and antioxidant production but not with lifespan, as was expected.

\section{Materials and Methods}

\section{Nematode Strains and Culture Conditions}

The Bristol N2 strain of Caenorhabditis elegans was used for all experiments. Nematodes were grown on $60 \mathrm{~mm}$ Petri plates containing NGM media with streptomycin at $20 \mu \mathrm{g} / \mathrm{mL}$ and seeded with OP50-1 Escherichia coli as a food source. Nematode and bacterial stocks were obtained from the Caenorhabditis Genetics Center (University of Minnesota, St. Paul, MN). Prior to experimentation, additional nematode stocks were stored at -80C following standard protocols (61) and thawed as needed for all assays. Worms were age-synchronized by standard bleach treatment prior to each assay and 
grown at either $20^{\circ} \mathrm{C}$ or $25^{\circ} \mathrm{C}$, as specified. Samples were acclimated to their respective temperature and food regimes for 2-3 generations prior to each experiment in an effort to minimize possible maternal effects.

Most of these assays were conducted using live E. coli as a nematode food source, which is standard practice in laboratory studies of C. elegans (Steirnagle 2011). I performed one life history assay at $25^{\circ} \mathrm{C}$ using live E. coli as a food source and one at 20 ${ }^{\circ} \mathrm{C}$ using UV-killed bacteria (see below for further detail). For any assay, NGM plates were seeded with $100 \mu \mathrm{L}$ OP50-1 E. coli that had been cultured at $37^{\circ} \mathrm{C}$ overnight in LB broth. Plates in the second life history assay $\left(20^{\circ} \mathrm{C}\right)$ were incubated at $37^{\circ} \mathrm{C}$ for 4 hours to grow a light bacterial lawn and were then UV sterilized (Logic Class II, Type A2 biosafety cabinet, Labconco, Kansas City, MO) for 2 hours at room temperature. Plates were stored overnight at room temperature and any plates with obvious signs of contamination, cracks or still-living OP50-1 colonies were discarded. Living colonies of OP50-1 can be easily identified by colony morphology after being allowed to sit overnight at room temperature.

\section{Paraquat Treatments}

Paraquat (PQ), also called methyl viologen dichloride, is an herbicide commonly used to experimentally generate exogenous oxidative stress by generating superoxide anions in vivo (10,56-58). For all assays in this study, NGM plates were supplemented with one of four PQ treatment levels: 0, 0.1, 1.0 and 2.0 mM PQ, hereafter referred to as control, low, medium and high treatment levels, respectively. These levels refer to the concentration of the PQ solutions rather than the final concentration in the media. Two 
hours prior to transferring worms, $0.5 \mathrm{~mL}$ of the appropriate $\mathrm{PQ}$ solution was added to each plate containing $15 \mathrm{~mL}$ solidified NGM and pre-seeded with OP50-1. PQ solutions were diluted in sterile water, which was also used as the control treatment. PQ solutions were stored at $4{ }^{\circ} \mathrm{C}$ covered in foil and made fresh prior to each assay. Nematodes were first exposed to their respective treatments on the unseeded plates following agesynchronization (as eggs). PQ treatment concentrations were decided upon in preliminary life history assays conducted using live $E$. coli which revealed that PQ levels of at least $5 \mathrm{mM}$ (stock) were required to induce a high proportion of larval arrest (data not shown). PQ levels that dramatically reduced or prevented the timely progression of worms through larval stages to reproductive maturity were not considered germane to this study.

\section{Temperature Treatments}

I conducted all preliminary assays and the first life history assay at $25^{\circ} \mathrm{C} \pm 2 \mathrm{C}$. This temperature is commonly used in the $C$. elegans literature $(14,47,53,62-65)$ as it allows for a faster generation time and reduced lifespan relative to $20^{\circ} \mathrm{C}$, which is another commonly used thermal regime in C. elegans studies. Twenty ${ }^{\circ} \mathrm{C}$ is considered to be the thermal optimum for $C$. elegans based on thermal preference experiments measuring intrinsic rate of growth (e.g., (66), although this species has broad thermal tolerance and exhibits remarkably weak preference for any particular temperature when allowed to explore a thermal gradient (67). I therefore repeated the life history and other assays at $20{ }^{\circ} \mathrm{C}$. The temperature at which each experiment was conducted is denoted in the results. 
E. coli Food Source

Food source type and temperature are noted for each assay as each is discussed.

\section{Life History Assays}

To determine the effects of the above treatments on fitness-related traits, two life history assays were performed following the general method of Estes et al. (2004). I conducted one life history assay using live E. coli at $25^{\circ} \mathrm{C}$ and a second trial at $20{ }^{\circ} \mathrm{C}$ using UV-killed E. coli (unless otherwise noted). Single L1 stage larvae were transferred to individual Petri plates. These focal nematodes were then transferred daily to fresh plates for at least five days following the first reproductive event to separate them from their progeny. However, for logistical reasons, worms were allowed to stay on plates for the remainder of their lives if no progeny were detected for the previous 24 hour period. Average total fecundity and reproductive schedules were obtained by counting surviving progeny on Toluidine-dye stained plates. Only worms that had successfully hatched from their eggs were counted. "Early fecundity" was calculated as the number of surviving offspring produced during the first and second days of plate transfer (which coincided with the first two days of reproduction in control worms), and "late fecundity" was the sum of the remaining days of reproduction. These traits capture the fitness effect of any change in the reproductive schedule caused by the stress treatments. Conversely, "relative early fecundity" and "relative late fecundity" describe only changes in fecundity and ignore changes in the reproductive schedule. Relative early fecundity was calculated as

the number of surviving offspring produced during the first two days of the reproductive period for each treatment, and relative late fecundity was calculated as the total number 
of offspring for any remaining days of reproduction. For example, if a worm produced 0 , 50,50 , and 0 offspring on the first, second, third and fourth days of transfer, respectively, its score for early fecundity would be 50 . Its score for late fecundity would also be 50 . However, the relative early and late fecundity for this worm would be 100 and 0 , respectively.

Total lifespan was calculated as number of days from L1 arrest until death. Worms were checked daily for survival and scored as dead if unresponsive to prodding with a platinum wire. Data were censored for nematodes that had desiccated after crawling onto the side of a plate.

Data from the life history assays described above were used to calculate relative fitness $(\omega)$. Relative fitness was calculated following the basic approach of Keightley et al. (2000, eq. 2) and further described by Estes et al. (2011). Specifically, I computed relative fitness for each individual as: $\omega=\Sigma e^{-r x} l(x) m(x)$, where $l(x)$ is the number of worms surviving to day $x$ and $m(x)$ is the fecundity at day $x$, and where $r$ is the mean intrinsic population growth rate of the control treatment worms from the appropriate assay. The latter was calculated by solving Euler's equation, $\omega e^{-r x} l(x) m(x)=1$, for $r$.

Finally, I quantified average correlations among the measured life history traits across PQ treatments to investigate whether the magnitude or pattern of these trait associations changed with increasing oxidative stress. 
Maturation Rate

In preparation for measuring mitochondrial oxidant levels as described below, one additional set of measurements was performed as part of the $20{ }^{\circ} \mathrm{C}$ and $25^{\circ} \mathrm{C}$ experiments and conducted using live E. coli. Time to first reproduction was assessed for control and PQ treated worms within each temperature regime by placing age synchronous L1 worms on individual plates and noting the hour at which the first egg(s) were produced. Beginning 30 hours after L1 arrest, nematodes experiencing $25{ }^{\circ} \mathrm{C}$ were surveyed hourly for eggs; hourly egg checks for nematodes experiencing $20^{\circ} \mathrm{C}$ began 50 hours after L1 arrest. The start time for these hourly time points were determined from preliminary experiments (data not shown). Each treatment cohort was removed for scoring from the incubator for identical lengths of time to control for differences in incubation and ambient temperature. Eggs were identified visually under a dissecting scope. Once the first eggs were identified, the parental hermaphrodite was sacrificed and the plate was allowed to incubate at room temperature to confirm egg viability the following day.

\section{Relative Mitochondrial Oxidant Levels}

For the $20^{\circ} \mathrm{C}$ and $25^{\circ} \mathrm{C}$ assays conducted using live E. coli, mitochondrial ROS levels were assessed using MitoSOX Red (Invitrogen, M36008, Carlsbad, CA) by following the basic methods developed by Dingley et al. (2010) and modified by Hicks, et al. (2012). Briefly, the terminal pharyngeal bulb was imaged in MitoSOX Red-labeled young adult nematodes from each treatment group. MitoSOX Red was recently shown to quantify total levels of mitochondrial oxidants, rather than superoxide specifically, when 
used with confocal microscopy (74). The terminal pharyngeal bulb was chosen for fluorescence imaging because it is an easily identified physical marker with a fluorescence level unaffected by the high dye uptake of lipid rich gut granules, and because the pharyngeal tissue is mitochondria-dense, remaining energetically active until senescence. Age synchronous worms were incubated for 24 hours in $10 \mu \mathrm{M}$ MitoSOX Red before being transferred as young adults to fresh NGM plates seeded with nonlabeled OP50-1 E. coli. They were allowed to feed for 1 hour, and paralyzed using a drop of $5 \mathrm{M}$ levamisole prior to imaging and $5 \mu \mathrm{L}$ polystyrene beads on agar pads (adapted from original methods developed by Christopher Fang-Yen, pers. comm.). 10 $\mu \mathrm{M}$ MitoSOX Red working solutions were prepared as part of the respective PQ treatments, which were made from a freshly prepared $5 \mathrm{M}$ MitoSOX Red stock solution in DMSO. The PQ + MitoSOX Red solution was added to plates as described above (Paraquat Treatments). Images of the pharynx were acquired using a high resolution wide field Core DV system (Applied Precision ${ }^{\mathrm{TM}}$ ), equipped with an Olympus IX71 inverted microscope mounted with a Nikon Coolsnap ES2 HQ camera (Advanced Light Microscopy Core Facility, Oregon Health and Science University, Portland, OR). Images were captured at $60 \mathrm{X}$ magnification with a 0.15 second exposure time and $5 \mu \mathrm{m} Z$-stack widths. Images were deconvolved prior to analysis. Terminal pharyngeal bulbs were manually encircled within each image and maximum pixel intensity was measured for this region within each z-stack slice using ImageJ software $(\mathrm{NIH})$. This procedure was applied to 40 exposed and 10 unexposed (control) animals for each PQ treatment. The final pharyngeal bulb intensity values were calculated as the difference between intensity values for exposed and control worms. Replicate maximum pixel value was calculated as 
the mean of all maximum pixel intensities. Finally, great care was taken to minimize technical error. For example, I exposed only one sample at a time to the microscope light source and used the shortest exposure time possible to avoid introducing variation due to breakdown of the dye.

Dyeing regimes in the $25^{\circ} \mathrm{C}$ experiment were applied at either the time of feeding after L1 arrest (24 hour trial), or 24 hours past feeding after L1 arrest (48 hr trial). Assay timing was adjusted in $20^{\circ} \mathrm{C}$ worms fed live $E$. coli as informed by the time to first reproduction results. Young worms were imaged 34 hours after L1 arrest and older worms were imaged 68 hours post L1 stage arrest. $25^{\circ} \mathrm{C}$ ROS data were log transformed to correct non-normal distributions.

\section{Statistical Analyses}

All statistical analysis was performed in JMP version 9.0.2 (SAS Statistical Inc., Cary, NC). In cases where data were non-normally distributed, attempts were made to normalize data through transformation. When transformations failed to normalize data for particular traits, these data were analyzed both by non-parametric analysis (Wilcoxon/Kruskal -Wallis tests using rank sums) and one-way analyses of variance (ANOVA) tests. When the results of parametric and non-parametric tests did not differ, parametric statistic and $p$ values are reported. These tests were performed to determine whether PQ treatment or temperature had a significant effect on individual life history traits. To determine whether traits differed among particular pairs of PQ treatment levels within each temperature regime, either the parametric Tukey-Kramer HSD test or the non-parametric Wilcoxon method was performed. Again, results of the parametric tests 
are reported when both types of tests gave the same result. Finally, I characterized associations among life history characters measured on the same individuals by calculating Spearman rank correlation coefficients between each pair of traits following Estes et al. (2011).

\section{Results}

Toward the major goal of assessing the effect of exogenous oxidative stress on life history in C. elegans, I assayed life history phenotypes and in vivo levels of ROS in worms treated with increasing concentrations of PQ and maintained in one of two experimental conditions: $25^{\circ} \mathrm{C}$ with a live $E$. coli food source and $20^{\circ} \mathrm{C}$ with UV-killed E. coli. A subset of these assays was also conducted at $20{ }^{\circ} \mathrm{C}$ with live E. coli. Results are reported for each measured phenotype assayed under these experimental conditions, which I denote as " $25^{\circ} \mathrm{C}$ " and " $20^{\circ} \mathrm{C}$ " below. It has been shown previously that unknown metabolite(s) of OP50 E. coli result in an innate immune response through the insulin-like IGF-1 signaling pathway which results in an almost 2-fold upregulation of sod-3 mRNA transcripts compared to nematodes grown in a monoxenic plated cultures of UV-killed OP50 (68). I reasoned that PQ's prooxidant nature probably results in the death or cessation of growth of E. coli which serves as a nematode food source, which would lead to a concomitant reduction in the aforementioned metabolite(s). In agreement with these expectations, I observed that bacterial lawns proliferated outside the original seeding location in the control and low PQ level treatment regime but showed minimal or no such proliferation in the medium and high PQ treated plates. I therefore chose to test whether similar results for life history traits would be observed if worms were fed only 
UV-killed OP50-1 by conducting a separate life history experiment. In this way, any effect that PQ treatments might have on the E. coli food source and any downstream effects on nematode life histories could be eliminated.

\section{Total Reproductive Output}

I counted individual daily progeny production for each worm in each PQ treatment to identify the effects of oxidative stress on reproductive output (fecundity) and the timing of the reproductive cycle.

$25{ }^{\circ} \mathrm{C}$

Worms treated with low and medium PQ concentrations experienced slightly increased reproductive output relative to control (no PQ) worms (Fig. 1A, Table 1). Animals in the low PQ treatment had more offspring $(205.7 \pm 6.0)$ than both the control (176.5 \pm 4.7$)$ and high PQ $(178.7 \pm 7.0)$ treatment level, but did not differ significantly from worms in the medium PQ treatment $(198.9 \pm 7.3)$ with respect to this trait (Wilcoxon $\mathrm{Z}=-0.754 \mathrm{p}=0.45$ ). Reproductive output in high PQ treatment animals was not statistically different from that in control animals (see Wilcoxon grouping Fig. 1A). Variance in total reproductive output among treatments did not differ significantly (Levene's test, $\mathrm{F}=1.41, \mathrm{p}=0.24$ ).

$20{ }^{\circ} \mathrm{C}$

In contrast to results from the $25^{\circ} \mathrm{C}$ conditions, total reproductive output in this assay showed a simpler, dose-dependent decline as PQ levels increased (Fig. 1B, Table 2). Low PQ treatment worms had slightly lower fecundity than control worms but did 
not differ significantly from the control treatment (Tukey-Kramer HSD, $\alpha=0.05$ ). While there was a statistically significant decline in total reproductive output observed in the medium PQ treatment and again in the high PQ treated worms (Fig. 1B, Table 2), I found no difference in variance among all treatments for total reproductive output (Levene's test, $\mathrm{F}=0.71, \mathrm{p}=0.55)$.

Reproductive Schedule

To evaluate the effects of oxidative stress on the schedule of reproduction, I measured daily progeny production for each nematode in each PQ treatment maintained in the aforementioned assay conditions.

$25^{\circ} \mathrm{C}$

Under normal lab conditions at $25^{\circ} \mathrm{C}$, the majority of $C$. elegans' reproductive output was achieved within a 48 hour period; however, an extended period of offspring production occurred over several additional days. Control treatment reproduction peaked on day 2 of reproductive period and declined to essentially zero by day 4 (Fig. 2A). Compared to the control, worms in the low and medium PQ treatments had near identical temporal patterns of reproduction while worms in the high PQ treatment experienced a delayed reproductive schedule (Fig. 2A). More specifically, worms in the low and medium PQ treatments had significantly more offspring on days 2 and 3 of reproduction while worms in the high PQ treatment had significantly fewer offspring on those days compared to the control (Tukey-Kramer HSD, $\alpha=0.05$ ). Peak reproduction occurred on day 2 for all worms in the assay, with the low PQ treatment worms having the most offspring (140.6 \pm 3.6$)$ followed by the medium treatment $(138.0 \pm 5.4)$, control 
treatment $(120.2 \pm 3.5)$, and worms in the high PQ treatment $(87.7 \pm 4.7)$. The third day saw a major ( $60 \%)$ decline in reproduction for all treatment levels except the high PQ treatment, which maintained a similar reproductive output to day 2. By the fourth day of reproduction, reproduction in the high PQ treated worms was still slightly elevated, but reproduction was negligible in all treatments and had completely ceased by day 5 (Fig. 2A).

$20{ }^{\circ} \mathrm{C}$

Similar to previous findings (22), control worms at $20^{\circ} \mathrm{C}$ followed a reproductive schedule that persisted for about 6 days with peak daily fecundity on day $3(60.4 \pm 2.8)$ (Fig. 2B). Low PQ treated worms had a similar schedule, yet a slight delay can be seen as low PQ treated worms had peak reproduction on day $4(61.3 \pm 2.6)$ rather than on day 3. Medium PQ treated worm reproduction also peaked on the fourth day of reproduction, although the peak was much lower than that of the other treatments $(41.2 \pm 2.7)$. The high PQ treated worms had low overall reproduction in addition to a markedly delayed reproductive schedule with all but one worm failing to produce offspring until the third day of reproduction in control worms. These high-PQ treated worms produced their peak number of offspring on the fifth day followed by a steady decline in offspring numbers. Notable is the large extension of the reproductive period for some high PQ treatment worms, with $\sim 25 \%$ of the surviving worms continuing to produce surviving offspring until the thirteenth day and the last offspring being produced on the $16^{\text {th }}$ day of the assay (after reproduction began for the control worms) (Fig. 2B). This pattern also diverges significantly from the $25^{\circ} \mathrm{C}$ assay reported above (Fig. 2A). 


\section{Maturation Rate}

To further characterize the effect of oxidative stress and temperature on the temporal pattern of reproduction, I measured the time at which worms produced their first surviving egg. This measurement will more precisely differentiate treatments in terms of their reproductive timing that the standard reproduction assays reported above. Further, I wished to know whether the $25^{\circ} \mathrm{C}$ temperature treatment might induce shock response pathways that confer ROS resistance, which could culminate in a PQ level-bytemperature interaction effect on reproductive timing. Note that both assays were conducted using live E. coli as a food source.

\section{$25{ }^{\circ} \mathrm{C}$}

I found that increasing PQ treatment level was associated with increasingly longer times to first reproduction (Fig. 3, leftmost data points). Although control and low-PQ treated worms both produced their first surviving eggs at approximately 42 hours after L1 arrest, nematodes in the medium PQ treatment were significantly delayed by 4.12 hours $( \pm 0.61)$ and worms in the high PQ treatment were delayed by 8.65 hours $( \pm 0.64)$ compared to the control. Additionally, while reproduction in the low-PQ treatment worms was not statistically different than that of the control, it was slightly delayed relative to control. This result is somewhat unexpected and indicates that the increase in total reproduction was not due to faster development to reproductive age.

\section{$20{ }^{\circ} \mathrm{C}$}

Although the average time to first reproduction was substantially longer at $20{ }^{\circ} \mathrm{C}$ as compared to $25^{\circ} \mathrm{C}(63.4 \pm 4.0$ vs. $42.4 \pm 2.8$ in control worms $)$, the pattern of 
differences among PQ treatments was almost identical at both temperatures (Fig. 3). At $20{ }^{\circ} \mathrm{C}$, medium-PQ treatment animals had a mean 4.3 hour $( \pm 0.48)$ delay in the amount of time to first egg lay, while high-PQ treatment worms had a 10.55 hour $( \pm 0.52)$ delay relative to controls.

A linear regression model testing the effects of temperature, PQ concentration, and the interaction of those two factors revealed that there were significant effects of both temperature and PQ concentration on mean time to egg laying, but no significant interaction effects between the two factors (Table 3). Visualizing the reaction norms in Fig. 3 highlight the essentially linear shift in mean time to first egg laid for all worms measured at each temperature. In other words no environment-by-environment interaction was observed for this trait.

\section{Lifespan}

I calculated mean lifespan for all cohorts in each treatment condition to determine how temperature and/or level of oxidative stress might modify this trait. I expected to find a general decrease in lifespan at the higher temperature $\left(25^{\circ} \mathrm{C}\right)$ as shown previously (22). I also hypothesized that mean lifespan would decline within temperature treatments as oxidative stress caused by paraquat increased in agreement with the oxidative stress theory of aging (11). $25^{\circ} \mathrm{C}$

There was no difference in mean lifespan among worms experiencing different PQ treatment levels (ANOVA F =0.49, p = 0.69; Fig. 4A). 
The resilience of mean lifespan to increasing oxidative stress was again evident at the lower temperature and on UV-killed OP50 E. coli food source. Mean lifespan did not differ among PQ treatment level (ANOVA F = 0.69, p = 0.56; Fig. 4B). Similarly, variance in lifespan also did not differ among treatments (Levene's test, $\mathrm{F}=0.44, \mathrm{p}=$ 0.72). A qualitative comparison of survival curves for all worms at each temperature is shown seen in Fig. 5.

Worms that died from bagging (premature hatching of eggs in the gonad resulting in death of the parent) were not removed from lifespan analysis. The relative few instances this occurred in each treatment could not be analyzed for variation among treatments or temperature due to small sample sizes.

\section{Relative Fitness}

Using the life history data reported above, I calculated relative fitness for each PQ treatment cohort for each assay (Fig. 6). Because ROS lead to many types of macromolecular damage, I expected that relative fitness should decline with increasing oxidative stress in both experimental conditions.

$25^{\circ} \mathrm{C}$

Worms subjected to the low PQ treatment level had the highest relative fitness (Fig. 6, Wilcoxon rank sum, $\alpha=0.05$ ) There was no statistical difference in pairwise mean comparisons between control and medium treatments. The cohort exposed to the highest level of PQ had significantly lower mean relative fitness compared to any of the 
lower PQ treatment levels even though mean fecundity was not statistically lower than the control group. Additionally, the variance in fitness of the medium-PQ treated worms was greater than that of the other groups (Levene's test, $F=3.91, p=0.0095$ ).

$20{ }^{\circ} \mathrm{C}$

Similar to the trend observed for total mean fecundity, worms incubated at $20^{\circ} \mathrm{C}$ and fed UV-killed E. coli had declining relative fitness as PQ levels increased (Fig. 6B). However, even though control and low PQ treatment worms had no statistically significant difference in total reproduction, they did have a distinct difference in relative fitness (Wilcoxon rank sum, $\alpha=0.05$ ). Finally, unlike the $25{ }^{\circ} \mathrm{C}$ assay, variance in fitness contracted with increasing oxidative stress (Levene's test, $F=9.44, p<0.001$, Fig. 6B).

\section{Correlations Among Life History Traits}

I compared life history traits with one another to determine whether predicted life history tradeoffs (negative correlations among fitness-related traits) would be revealed as stressful conditions increased.

$25{ }^{\circ} \mathrm{C}$

Figures 7 and 8 show average Spearman's pairwise rank correlations ( $\rho$ ) between life history traits for each oxidative stress level. Figure 7 reports correlations between the traits that are most informative with respect to testing the central hypothesis that oxidative stress mediates life history tradeoffs. Figure 8 reports associations between traits that are necessarily correlated with one another to some degree due to overlapping measurement (e.g., total reproduction and relative fitness) but which nonetheless reveal 
important information about the consequences of oxidative stress. Although many correlations tended to be negative in sign, there were very few statistically significant correlations $(\alpha=0.05)$ of either sign at any treatment level (see also Table 4). In fact, only two correlations were statistically distinguishable from zero. The first was between lifespan and late reproduction (see Methods) at the low PQ treatment level (Fig. 7). The second was a positive correlation between lifespan and early reproduction in the high-PQ treated cohort. In this trial, most of the significant correlations exist between the less informative pairs of traits (Fig. 8). This figure does, however, highlight the changing contributions of different life history components to relative fitness with increasing oxidative stress. For example, the correlation between relative fitness and lifespan, while zero in the other treatments, becomes significant and positive in high PQ treated worms $(\rho=0.32, p=0.022)$.

$20{ }^{\circ} \mathrm{C}$

Figures 9 and 10 show average Spearman's pairwise rank correlations ( $\rho$ ) between life history traits of worms grown at $20{ }^{\circ} \mathrm{C}$ and fed UV-killed bacteria and experiencing increasing levels of oxidative stress. In stark contrast to the previous assay, I observed a trend for life history correlations to become increasingly positive and statistically significant in the high PQ treatment cohort compared to the other treatment levels (Fig. 9). For this high-stress treatment, lifespan was significantly positively correlated with early reproduction $(\rho=0.68, p=0.0003)$ and with total reproduction $(\rho=$ $0.58, \mathrm{p}=0.004)$. Early and late reproduction are also correlated positively with each other $(\rho=0.63, p=0.0021)$. The only other statistically significant correlations appeared 
in the low PQ treatment between total reproduction and lifespan, which were positively related to one another $(\rho=0.36, p=0.02)$ and between early and late reproduction, which were negatively correlated $(\rho=-0.35, p=0.03)$.

Correlations between relative fitness and fitness-related life history traits revealed the expected positive associations (Fig. 10, Table 5). These findings are at least qualitatively similar to those of the $25^{\circ} \mathrm{C}$ assay apart from the negative correlation between lifespan and relative fitness observed for the control treatment (Fig. 10). Additionally, late reproduction appears to be a stronger contributor to overall fitness in this assay compared to the $25^{\circ} \mathrm{C}$ assay (Figs. 8 and 10).

\section{In Vivo ROS Levels}

In order to further understand how effectively these nematodes controlled ROS levels and how this affected the pattern of life history traits seen in previous assays, I measured in vivo ROS levels.

$25{ }^{\circ} \mathrm{C}$

To assess relative levels of ROS among oxidative stress treatments, we subjected worms to MitoSOX Red (Invitrogen) in their respective PQ treatments at either 24 or 48 hours to understand whether the pattern of ROS levels would be affected by age (see Methods). Dingley et al. (2010) measured relative fluorescence units (RFU) in nematodes exposed to a $24 \mathrm{hr} 1 \mathrm{mM} \mathrm{PQ}$ treatment, and found essentially equivalent RFU values for control and PQ treated worms. We expected to see similar patterns of RFU levels in our worms which were treated with much lower concentrations of PQ. Analysis of 
pharyngeal bulb fluorescence after 24 hours of PQ treatment revealed significant variation among oxidative stress treatment levels (ANOVA, $F=22.3, p<0.0001$ ) and statistically significant differences between every pair of treatments (Tukey HSD, $\alpha=$ 0.05) (Fig. 11A, filled symbols). ROS levels show an approximately linear decline from control levels to the low and medium PQ regimes and then increase slightly in the high PQ treatment level (control: $2.68 \pm 0.13$; low: $2.43 \pm 0.18$; medium: $2.10 \pm 0.20$; high: $2.27 \pm 0.21)$

In contrast to the above pattern, worms imaged at 48 hours after L1 arrest (i.e., young adults in the control treatment) showed a different pattern of ROS level with increasing oxidative stress (Fig. 11A, open symbols). Fluorescence levels in control worms were higher relative to the control cohort in the $24 \mathrm{hr}$ trial, although not significantly so $(t=0.53, p=0.699)$. ROS levels were higher at 48 hours than at 24 hours in both the low and medium PQ treatments, especially for the medium PQ treatment. Conversely, ROS levels were lower at 48 hours in the high PQ treated worms that for their 24 -hour trial counterparts $(2.27 \pm 0.04$ vs. $2.208 \pm 0.04$; Wilcoxon/ Kruskal-Wallis rank sum $Z=-3.90, \mathrm{p}<0.0001)$ (Fig. 11).

$20{ }^{\circ} \mathrm{C}$

I also quantified relative ROS levels (RFU) at $20^{\circ} \mathrm{C}$ in worms fed live E. coli (Fig. 11B). Unfortunately, a direct comparison cannot be drawn between ROS levels at $20{ }^{\circ} \mathrm{C}$ and $25^{\circ} \mathrm{C}$ as the filter set and lenses were changed in between assays; the trends relative to controls within each assay may, however, be confidently compared. For this assay, I captured fluorescent images of worms at 68 hours in an attempt to measure ROS 
at developmental stages similar to those in the $25^{\circ} \mathrm{C}$ assay; i.e., at 48 hours (see Methods). The 68 hour worms, a time at which the nematodes in the medium PQ treatment had (on average) developed into young adults, the trend in ROS values for 20 ${ }^{\circ} \mathrm{C}$ worms (Fig. 11B) is similar to $25^{\circ} \mathrm{C}$ worms at 48 hours (Fig. 11A, squares), although ROS levels in animals at the medium treatment are similar to the low treatment cohort and no longer higher than control worms.

\section{Discussion}

This thesis aimed to understand the physiological basis of life history tradeoffs by characterizing the effects of physiological stress on the interplay among fitness-related life history traits. I used laboratory studies with the model soil nematode, Caenorhabditis elegans, wherein animals were exposed to oxidative stress in the form of paraquat or a combination of oxidative and mild thermal stress. For animals in each treatment, I performed basic life tables to estimate fitness components and conducted fluorescence confocal microscopy to measure in vivo ROS levels. My central hypothesis was that increased levels of physiological stress would unmask life history tradeoffs predicted by classical theory.

\section{Nematode Oxidative and Thermal Stress Experiments}

There is a rich literature on oxidative stress-resistance in wildtype and mutant $C$. elegans genotypes. These assays almost always utilize acute and extreme oxidative insult while measuring the survival of focal nematodes in terms of hours or by assessing survival percentage after a few days $(47,49,50,52,53,57,64,75-82)$. These studies 
commonly use paraquat as the source of ROS, but some have used other ROS generators such as juglone $(53,80), \mathrm{H}_{2} \mathrm{O}_{2}$ (82), or sodium arsenite (81). When lower concentrations of prooxidants are used in lifespan assays, they are generally used in conjunction with 5fluoro-2'-deoxyuridine (FUdR) (50,63), which prevents mitotic cell division by inhibiting DNA synthesis. When applied to adult nematodes, FUdR simplifies lifespan assays by preventing oogenesis and thereby preventing reproduction (51). However, FUdR has also been shown to cause additional lifespan extension in two long-lived C. elegans mutants $(83,84)$, suggesting that the effects of this compound may not be fully understood. In any case, the trend of FUdR use has made assessment of fitness modification by oxidative or other stressors a rare occurrence indeed. Further, the use of FUdR encourages more brute-force attempts to assess resistance to unrealistic levels of oxidative stress. It is unlikely that acute oxidative stress is a common threat to $C$. elegans, which generally inhabits UV-free and hypoxic soil conditions (85). Since my research goal was to record how life history traits respond to biologically relevant levels of oxidative stress, I reasoned that a chronic exposure assay would be preferable.

To impose mild thermal stress on C. elegans populations, I chose to culture nematodes at $25^{\circ} \mathrm{C}$. Although thermal regime appears to be an issue of lab preference, much previous C. elegans research has been performed at $20{ }^{\circ} \mathrm{C}$ $(17,49,50,52,75,76,80,86-89)$, which is the accepted thermal optimum for N2 C. elegans in evolutionary studies (67). $25^{\circ} \mathrm{C} \pm 1 \mathrm{C}$ is also frequently used $(47,53,62-$ $65,75,78,90,91)$, perhaps primarily as a means to shorten the length of $C$. elegans life cycles. Culturing worms at higher temperatures reduces lifespan and mean total 
fecundity (22) and reduces maturation time (92) presumably through processes including a general increase in enzyme activities (93).

\section{Reproductive Traits}

The level of oxidative stress treatments at $25^{\circ} \mathrm{C}$ using live $E$. coli as a food source produced significant variation in reproductive traits (Fig. 1A; Table 1). Surprisingly a hormetic increase in total reproduction was found in worms exposed to low level oxidative insult relative to controls (Fig. 1A). This effect is interesting in light of the fact that self-reproductive capacity in these worms is known to be sperm limited (94). Total reproductive output declined slightly in medium PQ treated worms and further declined to control levels in high PQ treatment worms. A shift in the reproductive period effectively increased the generation time of the nematodes in the high PQ treatment (Fig. 3), however, and resulted in a significant reduction in their relative fitness when compared to controls (Fig. 6A). A previous study established that paraquat treatment caused a delay in maturation rate resulting in a shift in reproductive schedule without a reduction in fecundity (95), consistent with my findings. I hypothesize that this reproductive delay was caused by somatic maintenance, specifically the increased allocation of resources to oxidative stress response.

Compared to the $25{ }^{\circ} \mathrm{C}$ assay, reproductive output, timing, and relative fitness were more profoundly affected by paraquat treatment at $20^{\circ} \mathrm{C}$ using UV-killed E.coli as a food source. Total reproductive output showed a linear decline with increasing oxidative insult (Fig. 1B, Table 2). Combined with the severe delay in reproductive schedule that accompanied increasing oxidative stress (Fig. 2B), this reduced 
reproduction led to a sharp decline in relative fitness with increasing PQ treatment (Fig. $6 \mathrm{~B})$. Further, phenotypic variation among PQ treatment groups was elevated in worms fed UV-killed E. coli compared to those on the live E. coli diet; see F-ratios in Table 5 (UV-killed) versus Table 4 (live). The more pronounced effects of oxidative stress observed in this experiment may be from reduced SOD levels and/or dampened innate immune responses due to nematodes being fed a diet of UV-killed bacteria (see Materials and Methods). In support of the first idea, Jonassen et al, (88) found that $c l k-1$ mutants, which are unable to synthesize coenzyme $\mathrm{Q}\left(\mathrm{Q}_{9}\right)$, had arrested development unless their diet was supplemented with an alternative quinone, $\mathrm{Q}_{8}$, from OP50 E. coli. In addition to functioning as coenzymes within the mitochondrial ETC, quinones also complement the regular array of antioxidant molecules in vivo, functioning as lipid soluble free radical sinks in the cell (88). It is not known which other antioxidant molecules C. elegans obtains from its diet, but it is reasonable to suppose that nematodes in the $25^{\circ} \mathrm{C} /$ live $E$. coli experiment were acquiring an antioxidant buffer from their living bacterial food source whose own antioxidant systems were likely upregulated in response to the PQ treatment (96). In contrast, UV-killed E. coli are not producing proteins or metabolites that might assist antioxidant capacity in C. elegans exposed to paraquat. The current study is thus somewhat hobbled by the lack of a life history assay at $20^{\circ} \mathrm{C}$ using live bacteria, which would allow a direct assessment of the effects of food source on oxidative stress resistance in C. elegans without the possible confounding effects of temperature (see Chapter 3). However, a comparison of the maturation rate assays using live bacteria at both $20{ }^{\circ} \mathrm{C}$ and $25{ }^{\circ} \mathrm{C}$ (Fig. 3) and preliminary studies (data not shown) supported the idea that food source accounted for much of difference observed between assays in 
reproductive output in PQ-exposed worms. Specifically, control worms eating UV-killed E. coli developed much more slowly than those given live food, and a study at $20^{\circ} \mathrm{C}$ using live E. coli has shown that reproduction at this temperature is resistant to perturbation within the levels of oxidative stress tested here.

The shifts in reproductive timing observed with increasing PQ treatment observed in each assay (Fig. 3) may constitute a life history tradeoff within the framework of somatic maintenance prioritization over reproduction, because delaying the time to reproductive maturation can result in a major disadvantage for nematodes in terms of reproductive fitness (c.f., Hodgkin and Barnes 1991). Alternatively, delayed reproduction in times of stress could provide a fitness advantage if it allowed an organism to "wait out" the stressful conditions and produce offspring under more favorable conditions - even if the delay were accompanied by reduced total fecundity (98). Such a scenario is unlikely to explain the observations of this study since nematodes are not likely to encounter considerable levels of oxidative stress, fluctuating or otherwise, in nature. The pattern of delay in maturation rate observed with increasing levels of paraquat was not obscured by an interacting effect of temperature (Fig. 3; Table 3). As visualized in Fig. 3, PQ treatment and temperature each had significant effects on maturation rate, the latter of which produced an $\sim 20 \mathrm{hr}$ delay in maturation rate at $20{ }^{\circ} \mathrm{C}$ as compared to $25^{\circ} \mathrm{C}$; however, these factors did not interact in their effects on reproductive delay (Fig. 3). 
Lifespan

The mechanism(s) driving senescent decline, a universally documented condition among eukaryotes, is not currently known. Several factors like the accumulation of agecorrelated molecular aggregates (lipufuscin), decline in muscle mass, increase in genetic and protein damage, and loss of reproductive capability late in life have been linked to oxidative stress in C. elegans and have been used as evidence toward an oxidative stress theory of aging (43). In support of the oxidative stress theory of aging, resistance to prooxidants (along with resistance to temperature, UV irradiation, and hyperoxia) is a hallmark of long-lived mutants (87) possibly through upregulation of antioxidant defenses (76), although the biological relevance to certain of these stressors is an open question (85). I hypothesized that average lifespan would be negatively correlated with the level of oxidative insult in agreement with the oxidative stress theory of aging. Since ROS are known to damage to all macromolecules, lifespan should be one of the most obviously affected life history traits as this damage should accumulate with age (11). However, recent research suggests that an oxidative stress theory of aging may have been either an oversimplification or a case of causation attributed to strong correlation $(75,99$ 101). For example, Doonan, et al (49) found that RNAi knockdown of sod-2, a $C$. elegans gene coding for a cellular superoxide dismutase thought to provide a vital antioxidant function, resulted in higher levels of molecular oxidative damage, but no change in lifespan. In addition, I found no differences in nematode lifespan among PQ treatments in either life history trial (Fig. 4A and 4B), and variance in lifespan did not change significantly for either life history assay (Tables 4 and 5; Levene's test, $\alpha=0.05$ ) further chipping away at the oxidative stress theory of aging. These results are especially 
surprising when considering the life history assay at $20^{\circ} \mathrm{C}$ using UV-killed E. coli. PQ treatment caused substantial reductions in reproduction and relative fitness relative to control worms, yet lifespan was not negatively impacted with increasing oxidative insult. Reducing temperature increased lifespan, as expected, but had no interaction effect with oxidative treatment (Table 3, Fig. 5).

\section{Life History Tradeoffs}

Life history theory predicts that reproductive output and schedule should be significant determinants of lifespan $(4,18)$ and several studies have confirmed so-called 'costs of reproduction' $(20,25,102)$. For example, increased early reproduction should cause a reduction in lifespan owing to a genetic or physiological constraint. By the same token, if reproduction declines, there should be a positive response in the lifespan (98). Accordingly, long-lived age mutants in C. elegans are known to have delayed and reduced fecundity $(57,85,90,103,104)$, Within this context, I calculated the correlations among major life history traits in both assays. In contrast to the predicted tradeoffs, I found few significant correlations between lifespan and reproductive traits including total, early and late reproductive output (see Methods) for the $25^{\circ} \mathrm{C}$ assay (Table 4). Surprisingly, lifespan was negatively correlated with late reproduction in low PQ treated worms (Table 4, Fig. 7); lifespan and early reproduction were positively related in high PQ treated worms (Fig. 7). Both of these observations contrast with my initial predictions. Correlations between reproduction traits fitness were positive as expected due to the overlapping nature of those measurements. At $20{ }^{\circ} \mathrm{C}$, even stronger correlations were revealed, but they too were against predictions (Table 5, Figs. 9 and 
10). In fact, at $20^{\circ} \mathrm{C}$ the worms exposed to the highest level of $P Q$ had strongly positive correlations between reproductive output and lifespan. It is apparent that the simple model of a fixed total energy budget being divided between reproduction and somatic maintenance (c.f., Stearns, 1989) may be insufficient to describe the true nature of life history tradeoffs in this system.

The source of the strongly positive life history trait correlations observed in this study assays is unknown but could result from short-term evolutionary processes that occurred in our experiments. Paraquat should produce stochastic macromolecular damage through its production of superoxide anions $(12,55,105,106)$ and may therefore have generated genetic variation in our otherwise isogenic $C$. elegans populations. Since paraquat was applied to large populations of nematodes, genetic variants best able to endure the PQ treatments could have been enriched via selection within each starting population. Among these survivors, lifespan and reproduction were positively related. A direct test of this hypothesis would necessitate a study of the effects of the paraquat concentrations applied here on genomic stability.

\section{In Vivo ROS Levels}

I employed a method of in vivo ROS measurement established previously (72, 73). Previous results have shown ROS levels measured by fluorescent microscopy are similar to those measured in electron spin resonance assays (107). Additionally, ROS levels obtained via fluorescent microscopy from natural isolates of the congeneric nematode, C. briggsae, have been shown to correlate strongly with levels of oxidative DNA damage (J. Joyner Matos and S. Estes, unpubl. data). In the $25^{\circ} \mathrm{C}$ assay, ROS 
measurements in larval worms decline with PQ concentration and then increased slightly at the highest PQ treatment level - though not significantly beyond low PQ or control levels (Fig. 11A). This result indicates that larval worms are quite capable of suppressing ROS proliferation when treated with paraquat; the uptick in ROS at the highest PQ treatment level may indicate that a threshold to their antioxidant capacity is being approached. In accord with previous results showing that adult (or 'mixed stage') worms have reduced antioxidant capacity (60), low and medium PQ treatment cohorts had higher ROS levels at 48 hours (i.e., adult stage worms as determined via maturation rate assays) as compared to 24 hours (larval stage) (Fig. 11A). Worms tested after 48 hours in the high PQ level exhibited the lowest level of ROS, which was also lower than its larval stage counterparts (Fig. 11A). Maturation rate as modulated by PQ increase (Fig. 3) may provide an explanation for these findings; nematodes at the highest PQ treatment level are the slowest to mature to adulthood (high PQ, $51.0 \pm 4.3 \mathrm{hrs}$; control, $42.4 \pm 2.8 \mathrm{hrs}$ ) and might indicate that these worms are still in a larval stage and producing greater amounts of antioxidants to deal with the ROS generated by paraquat. This age difference as modulated by paraquat dosage might also then explain the difference in ROS measures between the medium and high PQ treated worms at 48 hours. Nematodes in the medium PQ treatment exhibited control levels of ROS (i.e., increased compared to that of high PQ treated worms) suggesting that antioxidant production may be reduced as these worms enter adulthood during this time.

ROS measurements of C. elegans raised at $20{ }^{\circ} \mathrm{C}$ and at a developmental time point analogous to $48 \mathrm{hrs}$ in the $25{ }^{\circ} \mathrm{C}$ assay ( taking into account the increased 
developmental time at $20^{\circ} \mathrm{C}$, Fig. 3) revealed a similar pattern of ROS level (compare Fig. 11A and 11B). Most notable is that the lowest ROS level was detected in the high PQ treated worms. The medium PQ cohort had higher levels of ROS relative to the high treatment as was the case at 48 hours, which provides further support for an agedependent modulation of antioxidant production during chronic stress. Here, however, ROS levels of medium PQ worms were lower than in control worms. This may reflect a reduction in the overall rate of endogenous ROS production at the lower temperature, but this was not tested.

While there were no quantifiable tradeoffs in the life history characters I tracked, these ROS data are strongly indicative of a tradeoff between reproductive fitness and antioxidant production. A previous study confirmed the upregulation of the antioxidant coding gene sod-3 in larval worms after having been exposed to paraquat (60). High treatment worms in this study had significantly lower ROS levels (Fig. 11), suggesting the upregulation of antioxidants with fitness levels reduced by over 40\% (Fig 6A), primarily through a delay in maturation (Fig 3). This suspected tradeoff was even more pronounced when the diet of C. elegans was changed to UV-killed bacteria (presumably reducing bacterial antioxidants available to the nematodes). Worms subjected to high levels of oxidative stress experienced a 13-fold reduction in relative fitness when compared to the control cohort.

\section{Summary}

This study provides support for the idea that somatic maintenance has priority over reproduction during periods of chronic oxidative and thermal stress. However, life 
history tradeoffs are not consistently manifested under stress in the fitness-related traits analyzed here. I have shown that reproductive traits are strongly negatively affected by oxidative stress, but that lifespan is entirely resilient to paraquat-derived oxidative. The latter finding provides evidence against the oxidative stress theory of aging. Further support for the resource allocation hypothesis comes from the finding that reproductive delays caused by oxidative stress treatment are accompanied by reduced ROS presumably through upregulation of antioxidant proteins. Finally, I provide evidence that the ability of $C$. elegans to withstand oxidative stress is related to its food source; $C$. elegans on a live-bacteria diet can mount a more effective response to ROS, through bacteria-derived metabolites and/or antioxidant molecules. 
Tables and Figures

Table 1. Life History Traits at $25^{\circ} \mathrm{C}$ on Live E. coli

\begin{tabular}{|c|c|c|c|c|c|}
\hline Trait & $P Q$ & Mean (SEM) & $n$ & F-ratio & p-value \\
\hline \multicolumn{6}{|c|}{ Total Reproduction } \\
\hline & Control & $176.52(6.14)$ & 56 & 5.4217 & 0.001 \\
\hline & Low & $205.72(6.25)$ & 54 & & \\
\hline & Medium & $198.91(6.31)$ & 53 & & \\
\hline & High & $178.71(6.37)$ & 52 & & \\
\hline \multicolumn{6}{|c|}{ Early Reproduction } \\
\hline & Control & $143.80(4.89)$ & 56 & 3.8095 & 0.01 \\
\hline & Low & 160.17 (4.98) & 54 & & \\
\hline & Medium & $160.59(5.03)$ & 53 & & \\
\hline & High & $166.19(5.08)$ & 52 & & \\
\hline \multicolumn{6}{|c|}{ Late Reproduction } \\
\hline & Control & $33.31(3.51)$ & 55 & 17.2848 & $<0.0001$ \\
\hline & Low & $47.92(3.64)$ & 51 & & \\
\hline & Medium & $39.06(3.61)$ & 52 & & \\
\hline & High & $12.52(3.61)$ & 52 & & \\
\hline \multicolumn{6}{|c|}{ Relative Fitness $(\omega)$} \\
\hline & Control & $1.02(0.03)$ & 56 & 39.7605 & $<0.0001$ \\
\hline & Low & $1.12(0.03)$ & 54 & & \\
\hline & Medium & $1.00(0.03)$ & 53 & & \\
\hline & High & $0.66(0.03)$ & 52 & & \\
\hline \multicolumn{6}{|l|}{ Lifespan } \\
\hline & Control & $12.71(0.53)$ & 56 & 0.4910 & 0.69 \\
\hline & Low & $13.19(0.54)$ & 54 & & \\
\hline & Medium & $13.19(0.55)$ & 53 & & \\
\hline & High & $12.40(0.55)$ & 52 & & \\
\hline
\end{tabular}

ANOVA tables for life history traits among PQ treatment levels (see methods for detailed treatment information) for nematodes at $25{ }^{\circ} \mathrm{C}$ on live OP50-1 E. coli. Standard error (SEM) is reported in parentheses following the respective mean for each trait within a treatment level.

Sample size (n) is listed in addition to F-ratio. P-value considered significant at $\alpha=0.05$. Degrees of freedom for all traits is 3 . 
Table 2. Life History Traits at $20^{\circ} \mathrm{C}$ on UV-killed E. coli

\begin{tabular}{|c|c|c|c|c|c|}
\hline Trait & $P Q$ & Mean (SEM) & $n$ & F-ratio & $p$-value \\
\hline \multicolumn{6}{|c|}{ Total Reproduction } \\
\hline & Control & $223.87(7.32)$ & 39 & 65.7856 & $<0.0001$ \\
\hline & Low & $209.37(7.42)$ & 38 & & \\
\hline & Medium & $148.32(7.52)$ & 37 & & \\
\hline & High & $71.04(9.54)$ & 23 & & \\
\hline \multicolumn{6}{|c|}{ Early Reproduction } \\
\hline & Control & $75.08(4.37)$ & 39 & 19.0686 & $<0.0001$ \\
\hline & Low & $73.13(4.43)$ & 38 & & \\
\hline & Medium & $53.57(4.49)$ & 37 & & \\
\hline & High & $26.57(5.69)$ & 23 & & \\
\hline \multicolumn{6}{|c|}{ Late Reproduction } \\
\hline & Control & $148.80(5.77)$ & 39 & 43.4533 & $<0.0001$ \\
\hline & Low & $136.24(5.84)$ & 38 & & \\
\hline & Medium & $94.76(5.92)$ & 37 & & \\
\hline & High & $48.71(7.86)$ & 21 & & \\
\hline \multicolumn{6}{|c|}{ Relative Fitness $(\omega)$} \\
\hline & Control & $1.05(0.04)$ & 39 & 77.8383 & $<0.0001$ \\
\hline & Low & $0.85(0.04)$ & 38 & & \\
\hline & Medium & $0.39(0.05)$ & 37 & & \\
\hline & High & $0.08(0.06)$ & 23 & & \\
\hline \multicolumn{6}{|l|}{ Lifespan } \\
\hline & Control & $23.62(1.09)$ & 39 & 0.6875 & 0.56 \\
\hline & Low & $22.13(1.11)$ & 38 & & \\
\hline & Medium & $24.30(1.12)$ & 37 & & \\
\hline & High & $22.91(1.42)$ & 23 & & \\
\hline
\end{tabular}

ANOVA tables for life history traits among PQ treatment levels (see methods for detailed treatment information) for nematodes at $20^{\circ} \mathrm{C}$ kept on UV-killed E. coli. Standard error (SEM) is reported in parentheses following the respective mean for each trait within a treatment level. Sample size (n) is listed in addition to F-ratio. P-value considered significant at $\alpha=0.05$. Degrees of freedom for all traits is 3 . 
Table 3. Results of ANOVA Model Analysis for Maturation Assays

\begin{tabular}{lcll}
\hline \multicolumn{1}{c}{ Source of Variation } & $d f$ & F-ratio & $p$-value \\
\hline Temperature & 1 & 2695.9 & $<0.0001$ \\
PQ Treatment & 3 & 110.8 & $<0.0001$ \\
Temperature $\times$ PQ Treatment & 3 & 0.95 & 0.42 \\
\hline Summary of two-way ANOVA for maturation rate assays conducted at $20{ }^{\circ} \mathrm{C}$ and \\
$25^{\circ} \mathrm{C}$. Variancein maturation rate depends on temperature regime and PQ level \\
but not on the interaction of these factors. & Both assays were performed using live \\
OP50-1 E. coli. &
\end{tabular}

Table 4. Spearman Pairwise Correlations and Individual Trait Variances at $25^{\circ} \mathrm{C}$ on live E. coli

\begin{tabular}{|c|c|c|c|c|c|c|}
\hline & & Early & Late & Total & Fitness & Lifespan \\
\hline \multirow{5}{*}{ 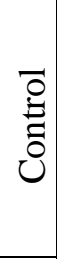 } & Early & 28.94 & -0.03 & 0.71 & 0.88 & 0.01 \\
\hline & Late & & 20.50 & 0.64 & 0.10 & -0.12 \\
\hline & Total & & & 35.40 & 0.71 & -0.08 \\
\hline & Fitness & & & & 0.20 & -0.01 \\
\hline & Lifespan & & & & & 4.14 \\
\hline \multirow{5}{*}{ ב } & Early & 27.67 & 0.02 & 0.74 & 0.91 & -0.08 \\
\hline & Late & & 27.09 & 0.74 & 0.16 & -0.31 \\
\hline & Total & & & 43.79 & 0.66 & -0.12 \\
\hline & Fitness & & & & 0.19 & -0.10 \\
\hline & Lifespan & & & & & 4.47 \\
\hline \multirow{5}{*}{ 导 } & Early & 41.12 & 0.03 & 0.64 & 0.70 & -0.11 \\
\hline & Late & & 35.87 & 0.70 & 0.49 & -0.13 \\
\hline & Total & & & 52.92 & 0.78 & -0.18 \\
\hline & Fitness & & & & 0.30 & -0.22 \\
\hline & Lifespan & & & & & 3.93 \\
\hline \multirow{5}{*}{$\begin{array}{l}\overrightarrow{0} \\
\stackrel{600}{10}\end{array}$} & Early & 46.09 & 0.04 & 0.84 & 0.82 & 0.28 \\
\hline & Late & & 16.76 & 0.44 & -0.17 & -0.05 \\
\hline & Total & & & 50.39 & 0.62 & 0.15 \\
\hline & Fitness & & & & 0.21 & 0.32 \\
\hline & Lifespan & & & & & 3.29 \\
\hline
\end{tabular}

PQ Treatment groupings on left, vertical. Individual trait variance given in diagonal (gray) for early, late and total reproduction, and fitness and lifespan. Statistically significant correlations are in bold $(\alpha=0.05)$. Data taken from life history assay at $25^{\circ} \mathrm{C}$ using live E. coli. 
Table 5. Spearman Pairwise Correlations and Individual Trait Variances at 20 ${ }^{\circ} \mathrm{C}$ on UV-killed E. coli

\begin{tabular}{|c|c|c|c|c|c|c|}
\hline & & Early & Late & Total & Fitness & Lifespan \\
\hline \multirow{5}{*}{ Ũ } & Early & 27.18 & $-2.6 \mathrm{E}-03$ & 0.61 & 0.64 & -0.24 \\
\hline & Late & & 31.90 & 0.72 & 0.39 & -0.01 \\
\hline & Total & & & 39.50 & 0.63 & -0.02 \\
\hline & Fitness & & & & 0.36 & -0.36 \\
\hline & Lifespan & & & & & 6.74 \\
\hline \multirow{5}{*}{ בـ } & Early & 31.87 & -0.35 & 0.39 & 0.38 & 0.07 \\
\hline & Late & & 41.79 & 0.65 & 0.19 & 0.29 \\
\hline & Total & & & 47.59 & 0.43 & 0.36 \\
\hline & Fitness & & & & 0.31 & 0.07 \\
\hline & Lifespan & & & & & 7.24 \\
\hline \multirow{5}{*}{$\stackrel{\Xi}{\Xi}$} & Early & 24.51 & 0.11 & 0.62 & 0.86 & -0.03 \\
\hline & Late & & 36.13 & 0.80 & 0.32 & -0.10 \\
\hline & Total & & & 46.46 & 0.71 & -0.03 \\
\hline & Fitness & & & & 0.20 & -0.13 \\
\hline & Lifespan & & & & & 6.84 \\
\hline \multirow{5}{*}{$\begin{array}{l}\frac{\pi}{50} \\
.00\end{array}$} & Early & 23.14 & 0.63 & 0.88 & 0.90 & 0.70 \\
\hline & Late & & 31.35 & 0.94 & 0.71 & 0.36 \\
\hline & Total & & & 51.05 & 0.89 & 0.58 \\
\hline & Fitness & & & & 0.09 & 0.68 \\
\hline & Lifespan & & & & & 6.16 \\
\hline
\end{tabular}

PQ Treatment groupings on left, vertical. Individual trait variance given in diagonal (gray) for early, late and total reproduction, and fitness and lifespan. Statistically significant correlations are in bold $(\alpha=0.05)$. Data taken from life history assay at $20^{\circ} \mathrm{C}$ using UV-killed E. coli. 
(A)

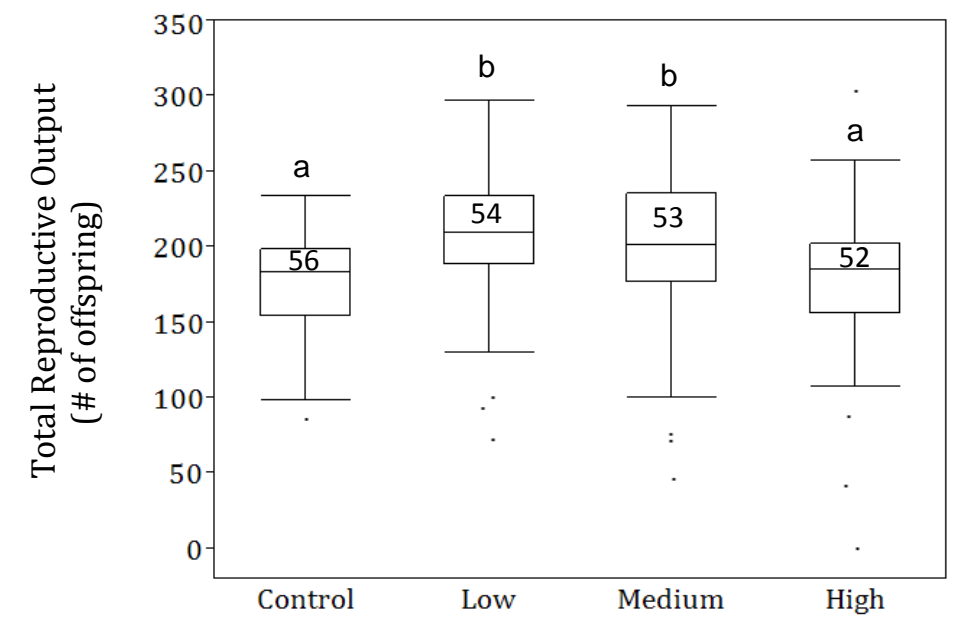

(B)

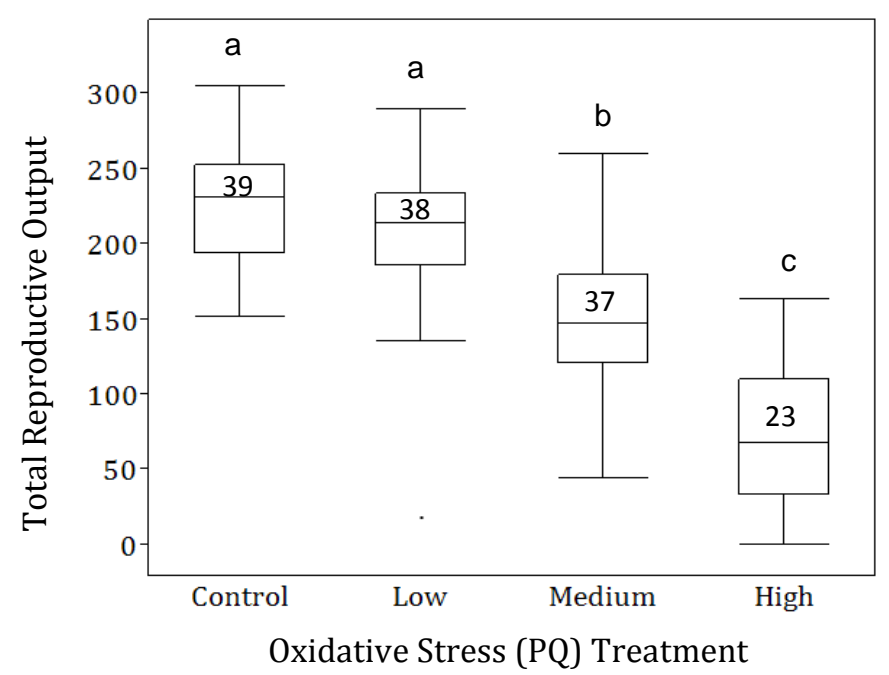

Figure 1. Total reproductive output in C. elegans is modified by temperature, oxidative stress, and diet. (A) Total fecundity increases at low and medium oxidative stress treatment levels and returns to control levels at the highest stress treatment level in worms fed live OP50-1 E. coli at $25^{\circ} \mathrm{C}$. (B) Total fecundity declines even with low levels of oxidative stress (NS) and continues to decline with increasing levels of stress in worms fed UV-killed E. coli at $20^{\circ} \mathrm{C}$. Letters above box plots group statistically identical means as indicated by pairwise Wilcoxon rank sum tests for non-parametric datasets $(\alpha=0.05)$ for panel A and Tukey-Kramer HSD, $\alpha=0.05$. Horizontal lines within boxes indicate the median while the top and bottom of the boxes bound the upper and lower quartiles, respectively. Whiskers denote the minimum and maximum values; outliers shown as dots. Sample sizes are indicated by numbers within the boxes. 

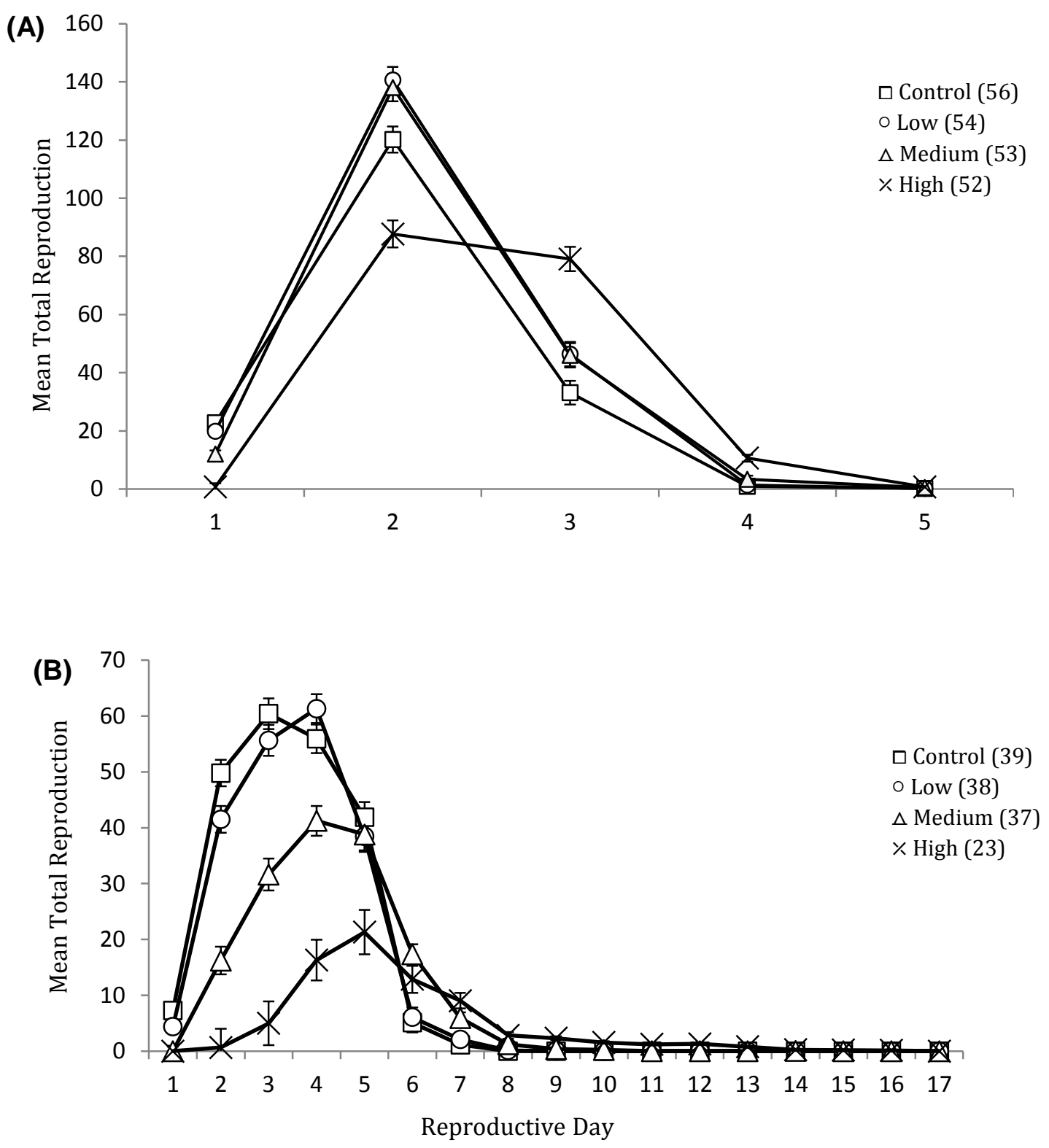

Figure 2. Reproductive schedules for each treatment level recorded as the mean number of surviving offspring per day of reproduction. Sample sizes listed parenthetically following legend entries. (A) Worms maintained at $25{ }^{\circ} \mathrm{C}$ with live OP50-1 E coli have similar reproductive schedules under control, low and medium oxidative stress treatments, while worms experiencing the highest stress level have a slightly delayed reproductive schedule. (B) Worms maintained at $20^{\circ} \mathrm{C}$ with UV-killed E. coli have lower overall reproductive output than worms experiencing $25^{\circ} \mathrm{C}$ and live $E$. coli (note change in scale between $\mathrm{A}$ and $\mathrm{B}$ panels) and show a reproductive delay even at the lowest oxidative stress treatment level, a pattern that is amplified under medium and high stress levels. Further, reproductive schedules for worms in all stress treatments were prolonged as compared to those in (A), but particularly for those experiencing the highest stress level. Each symbol represents mean reproductive output for corresponding day. Error bars represent one standard error (SEM). 


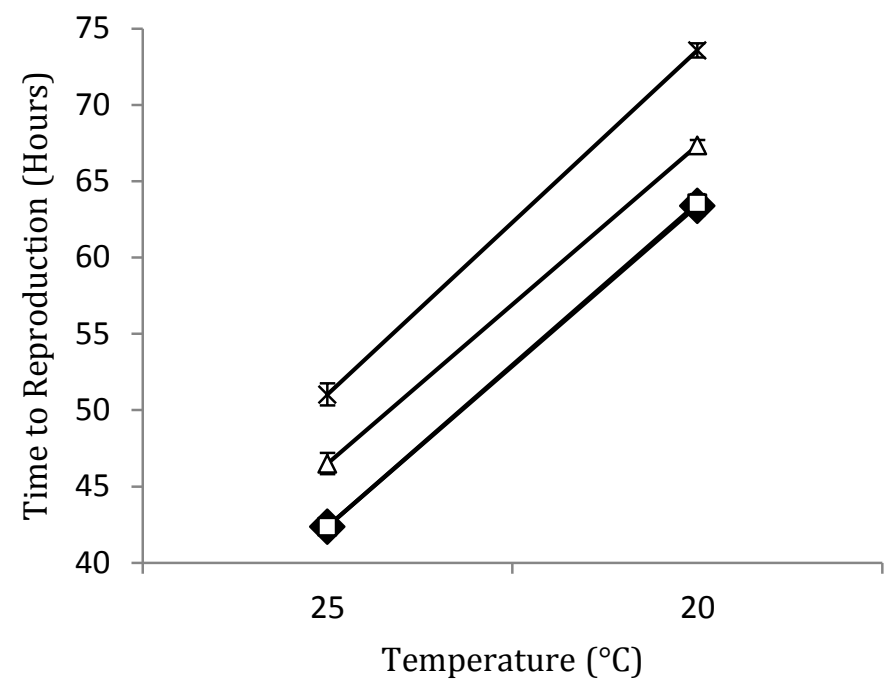

Figure 3. Time to reproduction determined by the hour of the first egg laid at control (squares) and three oxidative stress treatment levels (control= squares, low $=$ diamonds, medium $=$ triangles, high $=\mathrm{x}$; See Methods for details on treatment scheme) for worms raised under two temperature regimes and fed live E. coli. (Note that control data points overlap those for the low oxidative stress treatment at both temperatures.) 

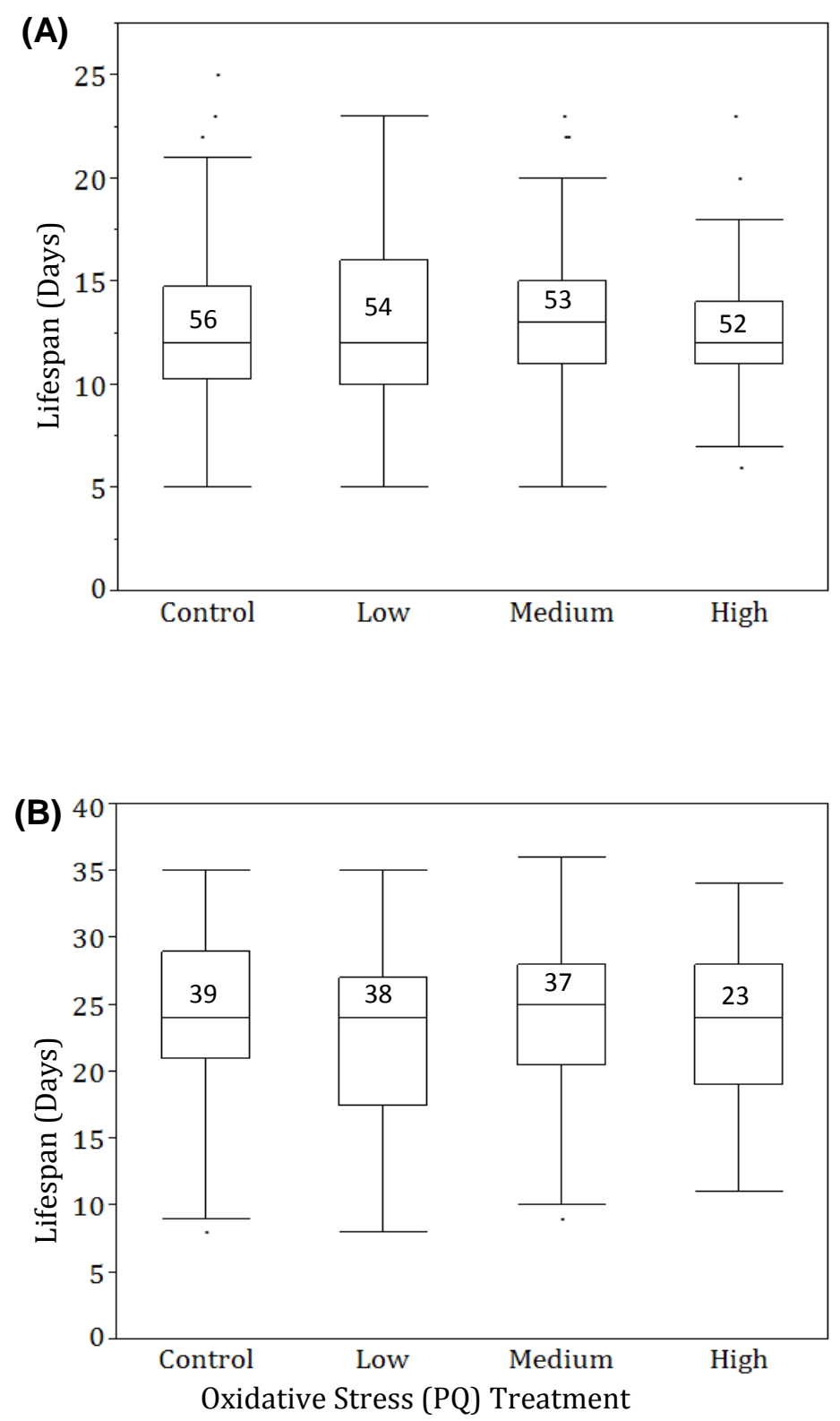

Figure 4. Mean lifespan as measured by total number of days lived beyond L1 stage arrest for worms experiencing (A) $25{ }^{\circ} \mathrm{C}$ and fed live OP50-1 E. coli. ANOVA testing indicated no significant variation among treatment means $(\mathrm{F}=0.49, \mathrm{p}=0.69)(\mathbf{B}) 20^{\circ} \mathrm{C}$ and fed UV-killed $E$. coli. No significant differences in mean lifespan were detected among oxidative stress treatment levels (ANOVA F $=0.69, \mathrm{p}=0.56$ ). However, worms in the second assay have increased mean lifespan as compared to the first (note change in scale between A and B panels; see Fig. 5 for details). Horizontal lines within boxes indicate the median while the top and bottom of the boxes bound the upper and lower quartiles, respectively. Whiskers denote the minimum and maximum values; outliers are shown as dots. Sample sizes are indicated by numbers within the boxes. 


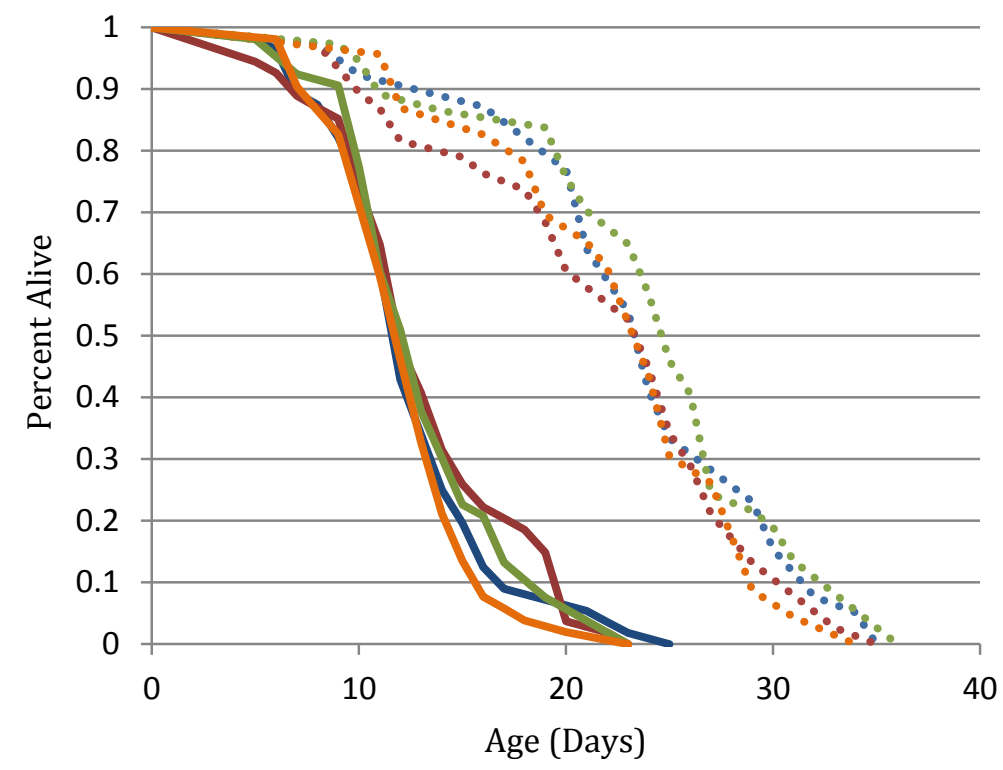

Figure 5. Survival curves for control worms raised at $25^{\circ} \mathrm{C}$ on live E. coli (solid) or on UV-killed E. coli at $20^{\circ} \mathrm{C}$ (dashed). PQ treatments: Control (blue), Low (red), Medium (green), High (orange). 

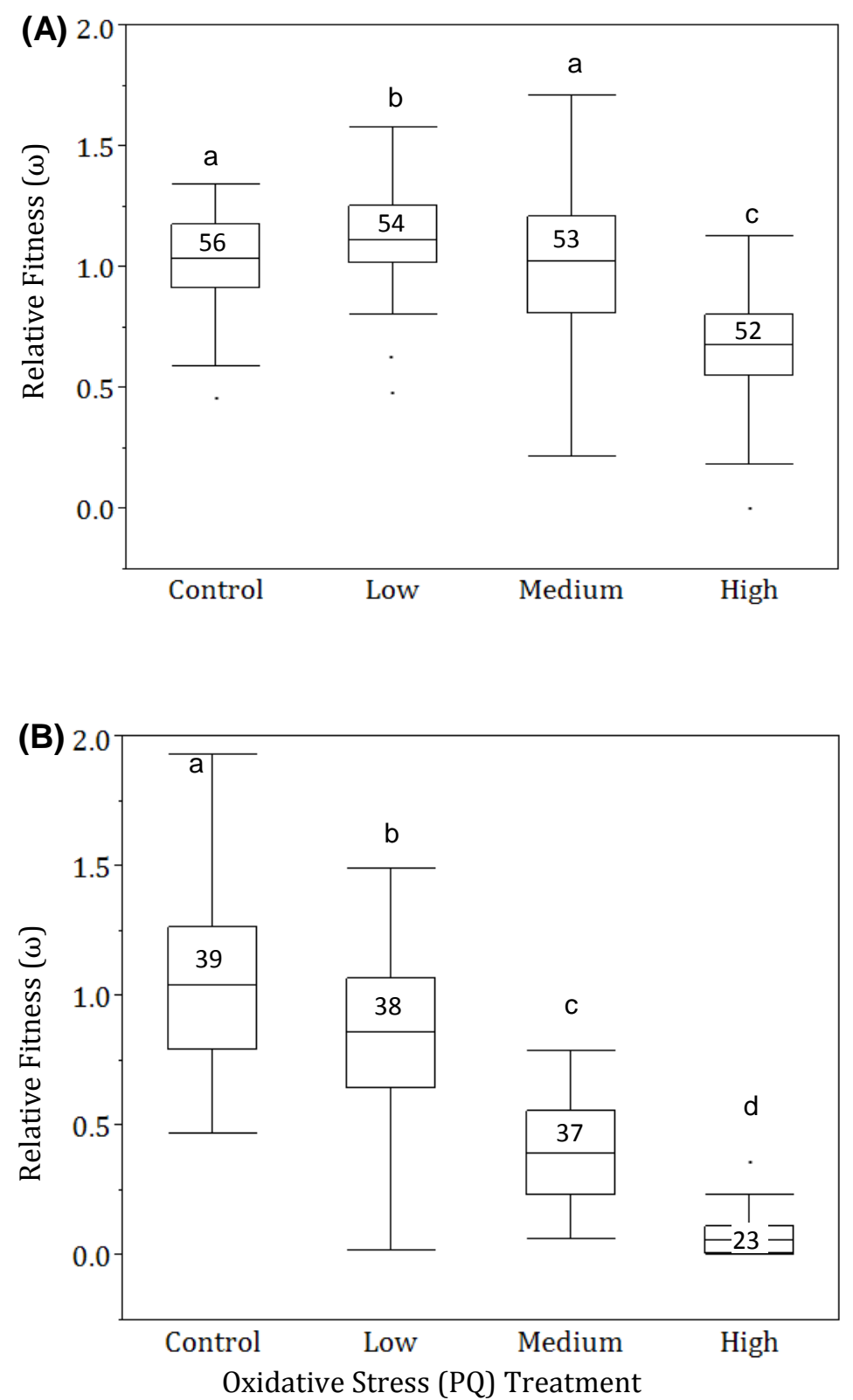

Figure 6. Relative fitness of worms experiencing (A) $25^{\circ} \mathrm{C}$ and fed live E. coli and (B) $20^{\circ} \mathrm{C}$ and fed UV-killed $E$. coli. Letters indicate statistically similar means as determined by pairwise Wilcoxon rank sum tests $(\alpha=0.05)$. Horizontal lines within boxes indicate the median while the top and bottom of the boxes bound the upper and lower quartiles, respectively. Whiskers denote the minimum and maximum values; outliers are shown as dots. Sample sizes are indicated by numbers within the boxes. 


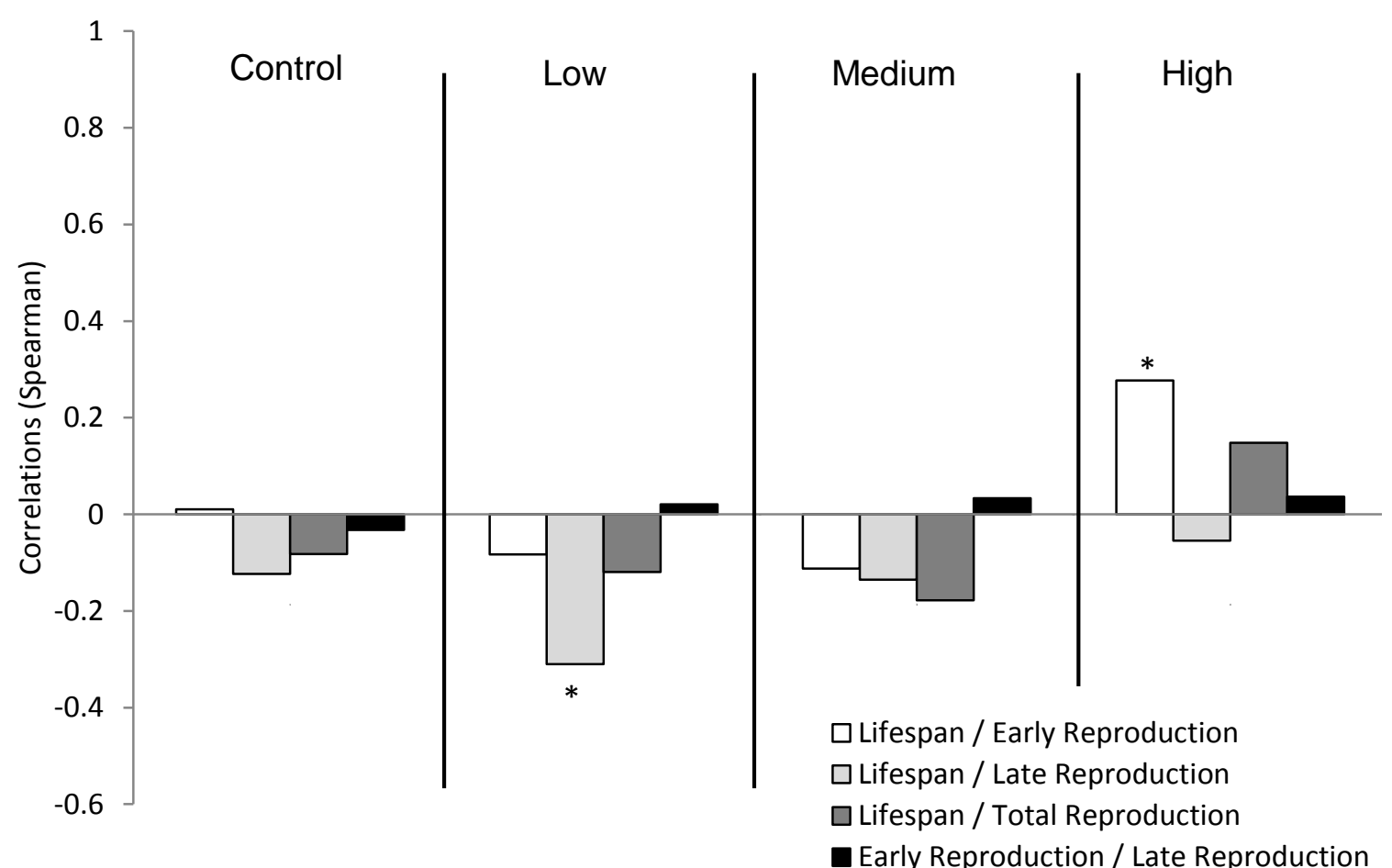

Figure 7. Spearman pairwise rank correlations for life history traits at each oxidative stress treatment level for worms grown at $25^{\circ} \mathrm{C}$ and fed live OP50-1 E. coli. Statistical significance is represented by asterisks where ${ }^{*}=\mathrm{p}<0.05$. 


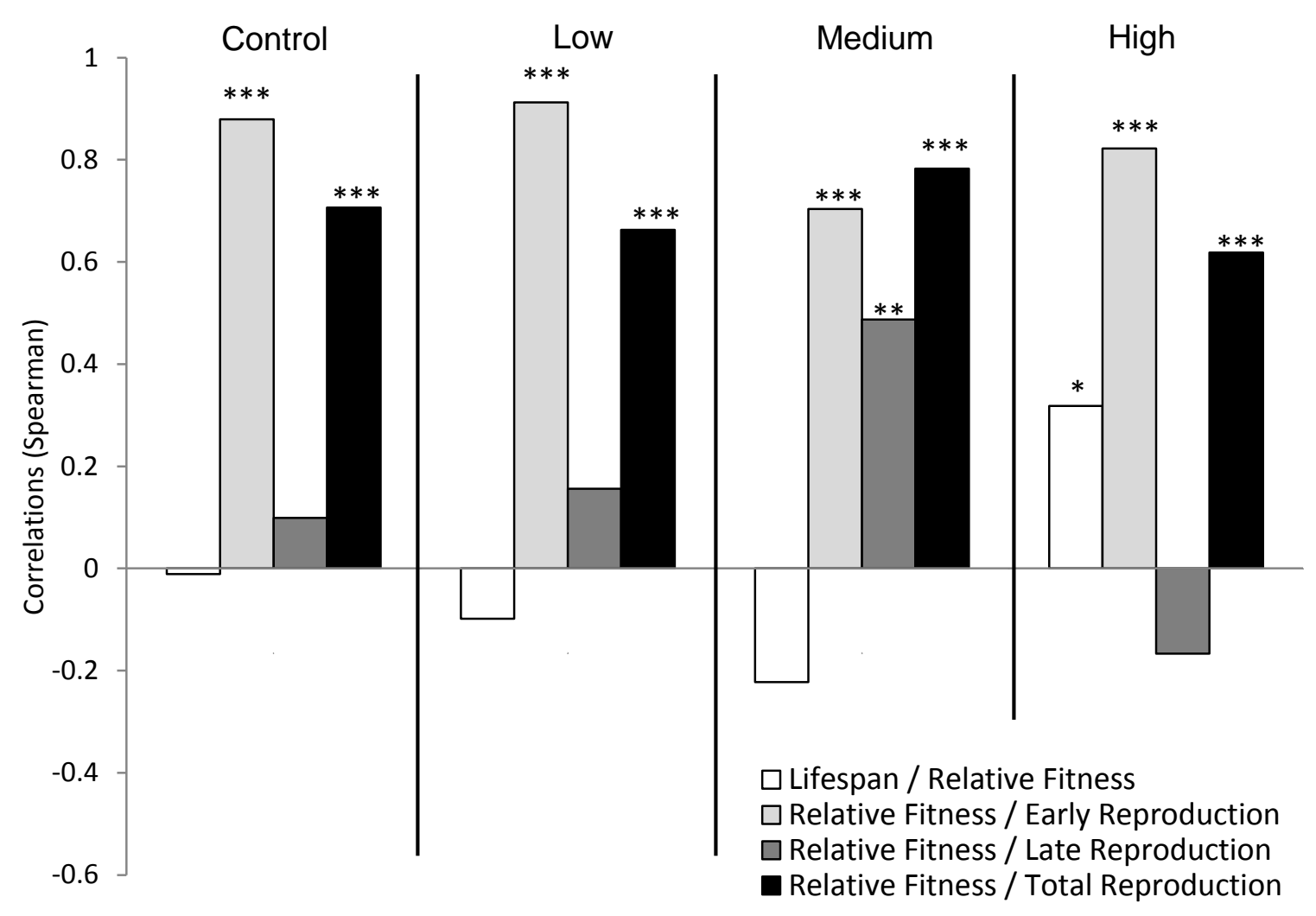

Figure 8. Spearman pairwise rank correlations for life history traits at each oxidative stress treatment level for worms grown at $25{ }^{\circ} \mathrm{C}$ and fed live OP50-1 E. coli. Statistical significance is represented by asterisks where $*=\mathrm{p}<0.05, * *=\mathrm{p}<0.001$ and $* * *=\mathrm{p}<0.0001$. 


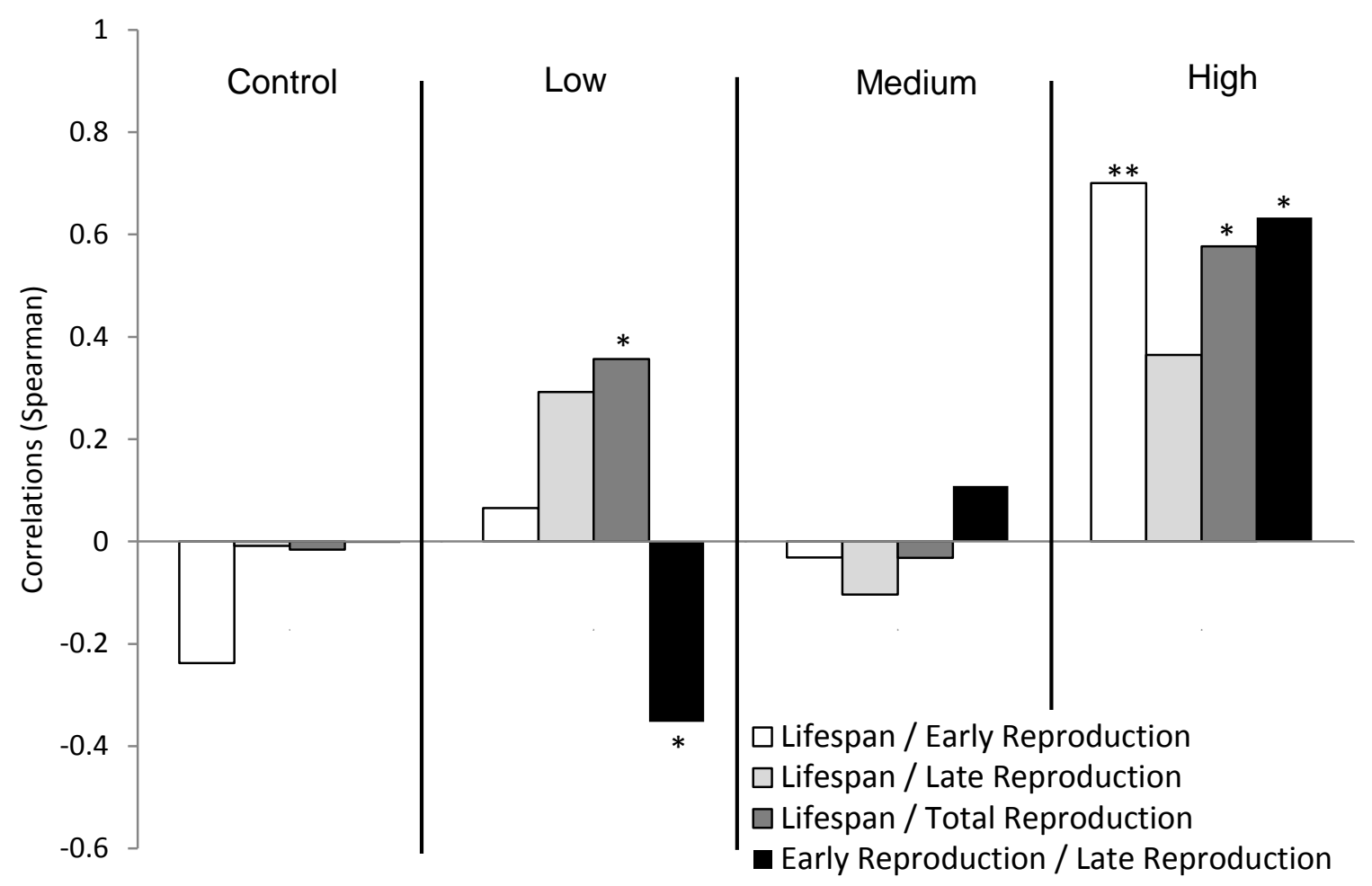

Figure 9. Spearman pairwise rank correlations for life history traits at each oxidative stress treatment level for worms grown at $20^{\circ} \mathrm{C}$ and fed UV-killed OP50-1 E. coli. Statistical significance is represented by asterisks where $*=\mathrm{p}<0.05, * *=\mathrm{p}<0.001$. 


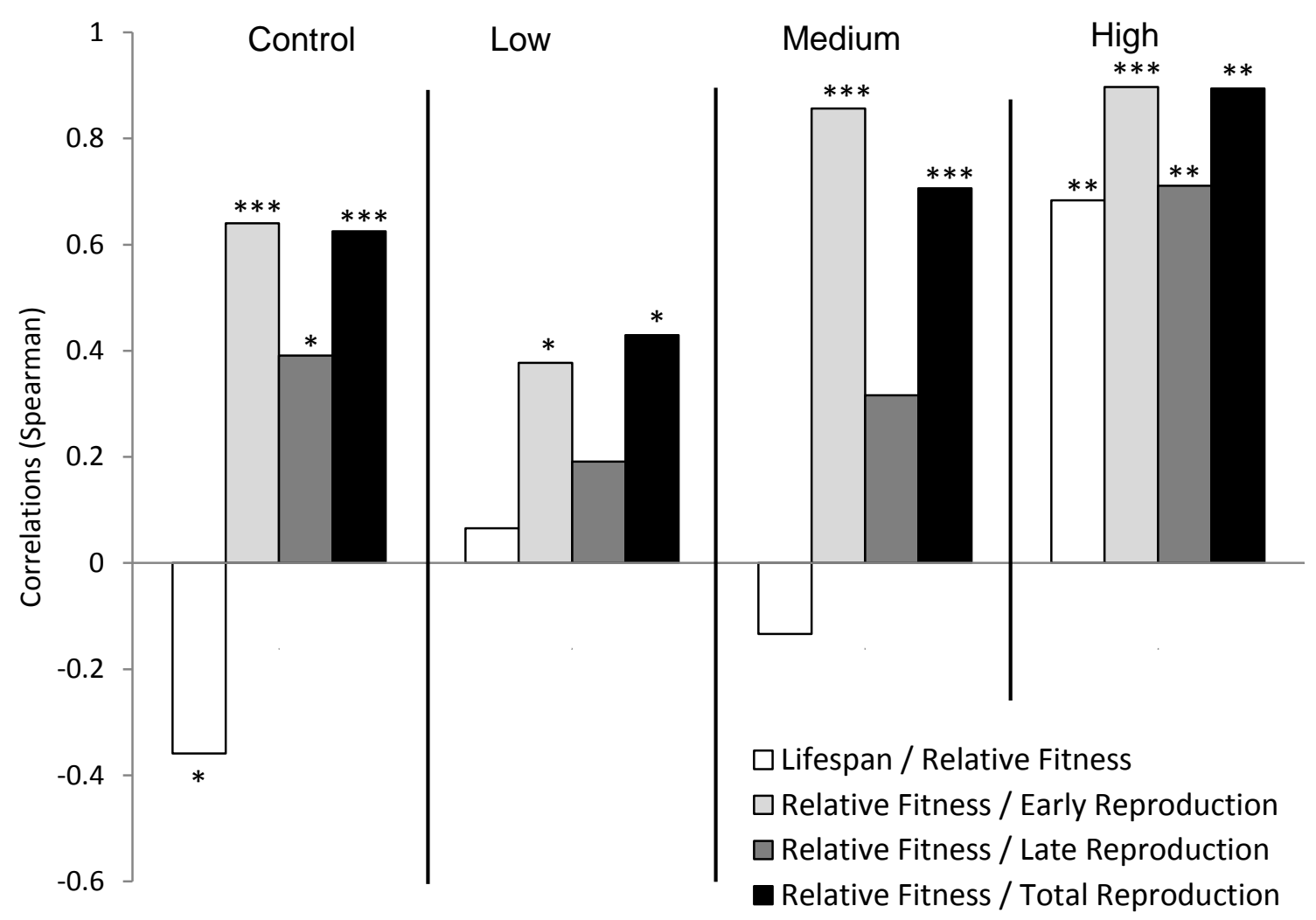

Figure 10. Spearman pairwise rank correlations for life history traits at each treatment level for worms grown at $20{ }^{\circ} \mathrm{C}$ and consuming UV-killed OP50-1. Statistical significance is given by number of asterisks as determined by p values; *: $\mathrm{p}<0.05 \& \geq 0.001, * *: \mathrm{p}<0.001 \& \geq 0.0001$., and ***: $\mathrm{p}$ $<0.0001$. 

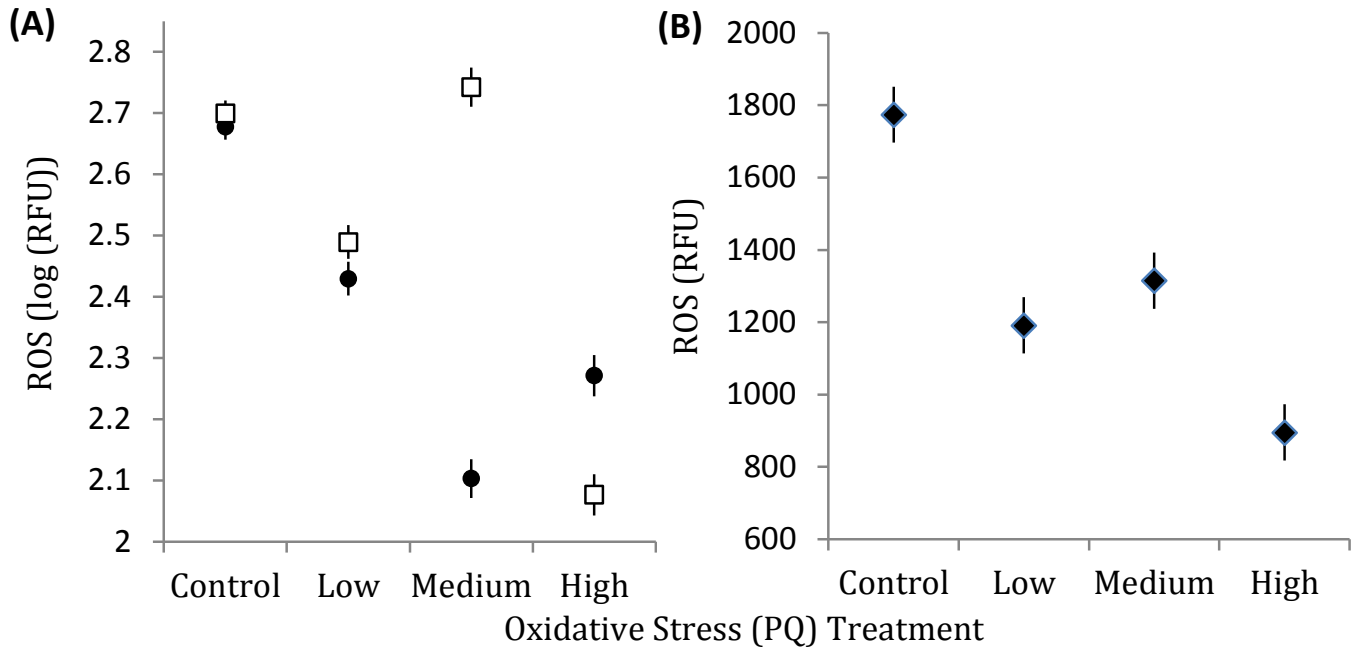

Figure 11. Reactive oxygen species (ROS) level measured as average maximum pixel intensity (RFU). (A) ROS levels in worms raised at $25{ }^{\circ} \mathrm{C}$ with live E. coli. Nematodes allowed to develop 24 hours (circles) and 48 hours (squares) after L1 arrest. Means log transformed to satisfy normality requirement of parametric tests. (B) ROS levels in worms allowed to develop for 68 hours (diamonds) raised at $20^{\circ} \mathrm{C}$ with live E. coli. Compare trend in data points to those in panel A (squares). See Figure 3 for more information on delay in time to reproduction. Error bars are 1 SEM in both panels. 
Chapter 3: Conclusions

In this study I showed that some fitness related life history traits of $C$. elegans, such as total reproductive output and maturation rate, respond negatively (with one notable exception) both to increasing levels of paraquat and to an increase in culture temperature. I also showed that reproductive output increased at low levels of paraquat in an apparent hormetic effect. In contrast, while lifespan was modified by temperature, a finding supported by previous researchers studies (22), lifespan did not vary in response to paraquat treatment level. The immutability of mean lifespan to the levels of oxidative stress used here provides support against an oxidative stress theory of aging. While quantitative tradeoffs on measured traits in this study were not found, the $44 \%$ relative fitness reduction and detected ROS levels were low in worms fed live E. coli in high paraquat treated worms during larval and adult stages suggests a tradeoff in terms of antioxidant control and reproduction exists in this system. To untangle the effects of food source, temperature, and oxidative stress on life history traits, I am currently performing a life history assay with live $E$. coli at $20^{\circ} \mathrm{C}$ as was previously completed at $25^{\circ} \mathrm{C}$. Additionally, I am in the process of measuring ROS levels in larval worms at $20{ }^{\circ} \mathrm{C}$ so as to compare with the same measurements taken at $25^{\circ} \mathrm{C}$. I hope to assess total antioxidant capacity by extending an oxygen radical absorbance capacity assay to this model system which should further elucidate the costs to reproduction in terms of antioxidant capacity in the near future. 


\section{References}

1. Reznick D, Nunney L, Tessier A. Big houses, big cars, superfleas and the costs of reproduction. Trends in Ecology \& Evolution. 2000;15(10):421-5.

2. Stearns SC. Life history evolution: successes, limitations, and prospects. Die Naturwissenschaften. 2000 Nov;87(11):476-86.

3. Plaistow SJ, Lapsley CT, Beckerman AP, Benton TG. Age and size at maturity: sex, environmental variability and developmental thresholds. Proceedings. Biological sciences / The Royal Society. 2004 May 7;271(1542):919-24.

4. Stearns SC. Trade-Offs in Life history Evolution. Functional Ecology. 1989;3(3):259-68.

5. Lack D. The Significance of Clutch-size. Ibis. 2008 Apr 3;89(2):302-52.

6. Houle D. Genetic covariance of fitness correlates: what genetic correlations are made of and why it matters. Evolution. 1991;45(3):630-48.

7. Brys K, Vanfleteren JR, Braeckman BP. Testing the rate-of-living/oxidative damage theory of aging in the nematode model Caenorhabditis elegans. Experimental Gerontology. 2007;42(9):845-51.

8. Cutler RG. Antioxidants and aging. The American journal of clinical nutrition. 1991 Jan;53(1 Suppl):373S-379S.

9. Harman D. Aging: a theory based on free radical and radiation chemistry. $\mathrm{J}$ Gerontol. 1956;11(3):298-300.

10. Gems D, Doonan R. Oxidative stress and ageing in the nematode Caenorhabditis elegans. Ageing Medicine: Oxidative Stress in Ageing: From Model Systems to Human Diseases. 2008;:81-110.

11. Beckman KB, Ames BN. The free radical theory of aging matures. Physiol Rev. 1998;78(2):547-81.

12. Monaghan P, Metcalfe NB, Torres R. Oxidative stress as a mediator of life history trade-offs: mechanisms, measurements and interpretation. Ecol Lett. 2009;12(1):75-92.

13. Speakman JR. Body size, energy metabolism and lifespan. The Journal of experimental biology. 2005 May;208(Pt 9):1717-30.

14. Riddle DL, Blumenthal T, Meyer BJ, Priess JR, editors. C. elegans II. 2nd ed. Cold Spring Harbor, NY: Cold Spring Harbor Laboratory Press; 1997.

15. Leung MCK, Williams PL, Benedetto A, Au C, Helmcke KJ, Aschner M, et al. Caenorhabditis elegans: an emerging model in biomedical and environmental toxicology. Toxicological sciences : an official journal of the Society of Toxicology. 2008 Nov;106(1):5-28.

16. Johnson TE. Caenorhabditis elegans 2007: the premier model for the study of aging. Experimental gerontology. 2008 Jan;43(1):1-4.

17. Gems D, Riddle DL. Genetic, behavioral and environmental determinants of male longevity in Caenorhabditis elegans. Genetics. 2000 May;154(4):1597-610. 
18. Zera AJ, Harshman LG. The Physiology of Life History Trade-Offs in Animals. Annual Review of Ecology and Systematics. 2001;32(1):95-126.

19. Blount JD. Carotenoids and life history evolution in animals. Arch Biochem Biophys. 2004;430(1):10-5.

20. Alonso-Alvarez C, Bertrand S, Devevey G, Prost J, Faivre B, Sorci G. Increased susceptibility to oxidative stress as a proximate cost of reproduction. Ecology Letters. 2004;7(5):363-8.

21. Alonso-Alvarez C, Bertrand S, Devevey G, Prost J, Faivre B, Chastel O, et al. An experimental manipulation of life history trajectories and resistance to oxidative stress. Evolution. 2006;60(9):1913-24.

22. Klass MR. Aging in the nematode Caenorhabditis elegans: major biological and environmental factors influencing life span. Mech Ageing Dev. 1977;6(6):413-29.

23. Roff DA, Fairbairn DJ. The evolution of trade-offs: where are we? Journal of evolutionary biology. 2007 Mar;20(2):433-47.

24. Van Noordwijk AJ, De Jong G. Acquisition and allocation of resources: their influence on variation in life history tactics. The American Naturalist. 1986;128(1):137-42.

25. Wang Y, Salmon AB, Harshman LG. A cost of reproduction: oxidative stress susceptibility is associated with increased egg production in Drosophila melanogaster. Exp Gerontol. 2001;36(8):1349-59.

26. Chippindale AK, Chu TJF, Rose MR. Complex Trade-Offs and the Evolution of Starvation Resistance in Drosophila melanogaster. Evolution. 1996;50(2):753-66.

27. Dowling DK, Simmons LW. Reactive oxygen species as universal constraints in life history evolution. Proc R Soc B. 2009;276:1737-45.

28. Pearl R. The rate of living. London: University of London Press; 1928.

29. Perez-Campo R, López-Torres M, Cadenas S, Rojas C, Barja G. The rate of free radical production as a determinant of the rate of aging: evidence from the comparative approach. Journal of comparative physiology. B, Biochemical, systemic, and environmental physiology. 1998 Apr;168(3):149-58.

30. Murphy MP. How mitochondria produce reactive oxygen species. Biochemical Journal. 2009;417:1-13.

31. Klass M, Nguyen PN, Dechavigny A. Age-correlated changes in the DNA template in the nematode Caenorhabditis elegans. Mechanisms of Ageing and Development. 22(3-4):253-63.

32. Richter C, Park JW, Ames BN. Normal oxidative damage to mitochondrial and nuclear DNA is extensive. Proc Natl Acad Sci U S A. 1988;85(17):6465-7.

33. Beckman KB, Ames BN. Oxidative decay of DNA. J Biol Chem. 1997;272(32):19633-6.

34. Anson R, Bohr V. Mitochondria, oxidative DNA damage, and aging. 2000;(4):199-218.

35. Hartman PS, Ishii N, Kayser EB, Morgan PG, Sedensky MM. Mitochondrial mutations differentially affect aging, mutability and anesthetic sensitivity in Caenorhabditis elegans. Mech Ageing Dev. 2001;122(11):1187-201.

36. Finkel T, Holbrook NJ. Oxidants, oxidative stress and the biology of ageing. Nature. 2000 Nov 9;408(6809):239-47. 
37. Bergeron P, Careau V, Humphries MM, Réale D, Speakman JR, Garant D. The energetic and oxidative costs of reproduction in a free-ranging rodent. Functional Ecology. 2011 Oct 18;25(5):1063-71.

38. Garratt M, Vasilaki A, Stockley P, McArdle F, Jackson M, Hurst JL. Is oxidative stress a physiological cost of reproduction? An experimental test in house mice. Proceedings. Biological sciences / The Royal Society. 2011 Apr 7;278(1708):1098-106.

39. Kim S-Y, Noguera JC, Morales J, Velando A. Quantitative genetic evidence for trade-off between growth and resistance to oxidative stress in a wild bird. Evolutionary Ecology. 2010 Sep 10;25(2):461-72.

40. Mougeot F, Martínez-Padilla J, Blount JD, Pérez-Rodríguez L, Webster LMI, Piertney SB. Oxidative stress and the effect of parasites on a carotenoid-based ornament. The Journal of experimental biology. 2010 Feb 1;213(3):400-7.

41. Metcalfe NB, Alonso-Alvarez C. Oxidative stress as a life history constraint: the role of reactive oxygen species in shaping phenotypes from conception to death. Functional Ecology. 2010 Oct 30;24(5):984-96.

42. Pamplona R, Barja G. An evolutionary comparative scan for longevity-related oxidative stress resistance mechanisms in homeotherms. Biogerontology. 2011 Oct;12(5):409-35.

43. Van Raamsdonk JM, Hekimi S. Reactive Oxygen Species and Aging in Caenorhabditis elegans: Causal or Casual Relationship? Antioxid Redox Signal. 2010;13(12):1911-53.

44. Feng JL, Bussiere F, Hekimi S. Mitochondrial electron transport is a key determinant of life span in Caenorhabditis elegans. Developmental Cell. 2001;1(5):633-44.

45. Tzou P, De Gregorio E, Lemaitre B. How Drosophila combats microbial infection: a model to study innate immunity and host-pathogen interactions. Current Opinion in Microbiology. 2002;5(1):102-10.

46. Valko M, Leibfritz D, Moncol J, Cronin MTD, Mazur M, Telser J. Free radicals and antioxidants in normal physiological functions and human disease. The International Journal of Biochemistry \&amp; Cell Biology. 2007;39(1):44-84.

47. Yen K, Patel HB, Lublin AL, Mobbs CV. SOD isoforms play no role in lifespan in ad lib or dietary restricted conditions, but mutational inactivation of SOD-1 reduces life extension by cold. Mechanisms of ageing and development. 2009 Mar;130(3):173-8.

48. Van Raamsdonk JM, Hekimi S. Deletion of the Mitochondrial Superoxide Dismutase sod-2 Extends Lifespan in Caenorhabditis elegans. Plos Genetics. 2009;5(2).

49. Doonan R, McElwee JJ, Matthijssens F, Walker GA, Houthoofd K, Back P, et al. Against the oxidative damage theory of aging: superoxide dismutases protect against oxidative stress but have little or no effect on life span in Caenorhabditis elegans. Genes \& development. 2008 Dec 1;22(23):3236-41.

50. Pun PB, Gruber J, Tang SY, Schaffer S, Ong RL, Fong S, et al. Ageing in nematodes: do antioxidants extend lifespan in Caenorhabditis elegans? Biogerontology. 2010;11(1):17-30. 
51. Gruber J, Ng LF, Poovathingal SK, Halliwell B. Deceptively simple but simply deceptive--Caenorhabditis elegans lifespan studies: considerations for aging and antioxidant effects. FEBS Lett. 2009;583(21):3377-87.

52. Keaney M, Matthijssens F, Sharpe M, Vanfleteren J, Gems D. Superoxide dismutase mimetics elevate superoxide dismutase activity in vivo but do not retard aging in the nematode Caenorhabditis elegans. Free radical biology \& medicine. 2004 Jul 15;37(2):239-50.

53. Burmeister C, Luersen K, Heinick A, Hussein A, Domagalski M, Walter RD, et al. Oxidative stress in Caenorhabditis elegans: protective effects of the Omega class glutathione transferase (GSTO-1). FASEB J. 2008;22(2):343-54.

54. Yang W, Tiffany-Castiglioni E. The bipyridyl herbicide paraquat produces oxidative stress-mediated toxicity in human neuroblastoma SH-SY5Y cells: relevance to the dopaminergic pathogenesis. J Toxicol Environ Health A. 2005;68(22):1939-61.

55. Bus JS, Aust SD, Gibson JE. Paraquat toxicity: proposed mechanism of action involving lipid peroxidation. Environmental health perspectives. 1976 Aug; 16:139-46.

56. Hassan HM, Fridovich I. Intracellular production of superoxide radical and of hydrogen peroxide by redox active compounds. Arch Biochem Biophys. 1979;196(2):385-95.

57. Ishii N, Takahashi K, Tomita S, Keino T, Honda S, Yoshino K, et al. A methyl viologen-sensitive mutant of the nematode Caenorhabditis elegans. Mutation Research/DNAging. 1990;237(3-4):165-71.

58. Winterbourn CC. Reconciling the chemistry and biology of reactive oxygen species. Nat Chem Biol. 2008;4(5):278-86.

59. Dillin A, Hsu AL, Arantes-Oliveira NA, Lehrer-Graiwer J, Hsin H, Fraser AG, et al. Rates of behavior and aging specified by mitochondrial function during development. Science. 2002;298(5602):2398-401.

60. Tawe WN, Eschbach ML, Walter RD, Henkle-Duhrsen K. Identification of stressresponsive genes in Caenorhabditis elegans using RT-PCR differential display. Nucleic Acids Res. 1998;26(7):1621-7.

61. Stiernagle T. Maintenance of C. elegans. In: The C. elegans Research Community, editor. WormBook. WormBook; 2011.

62. Garigan D, Hsu A-L, Fraser AG, Kamath RS, Ahringer J, Kenyon C. Genetic analysis of tissue aging in Caenorhabditis elegans: a role for heat-shock factor and bacterial proliferation. Genetics. 2002 Jul 1;161(3):1101-12.

63. Lee GD, Wilson MA, Zhu M, Wolkow CA, De Cabo R, Ingram DK, et al. Dietary deprivation extends lifespan in Caenorhabditis elegans. Aging Cell. 2006;5(6):515-24.

64. Lee H, Cho JS, Lambacher N, Lee J, Lee SJ, Lee TH, et al. The Caenorhabditis elegans AMP-activated protein kinase AAK-2 is phosphorylated by LKB1 and is required for resistance to oxidative stress and for normal motility and foraging behavior. J Biol Chem. 2008;283(22):14988-93. 
65. TeKippe M, Aballay A. C. elegans germline-deficient mutants respond to pathogen infection using shared and distinct mechanisms. PloS one. 2010 Jan;5(7):e11777.

66. Anderson JL, Albergotti L, Ellebracht B, Huey RB, Phillips PC. Does thermoregulatory behavior maximize reproductive fitness of natural isolates of Caenorhabditis elegans? BMC evolutionary biology. 2011 Jan;11:157.

67. Anderson JL, Albergotti L, Proulx S, Peden C, Huey RB, Phillips PC. Thermal preference of Caenorhabditis elegans: a null model and empirical tests. The Journal of experimental biology. 2007 Sep;210(Pt 17):3107-16.

68. Hahm J-H, Kim S, Paik Y-K. GPA-9 is a novel regulator of innate immunity against Escherichia coli foods in adult Caenorhabditis elegans. Aging Cell. 2011;10(2):208-19.

69. Estes S, Phillips PC, Denver DR, Thomas WK, Lynch M. Mutation accumulation in populations of varying size: the distribution of mutational effects for fitness correlates in Caenorhabditis elegans. Genetics. 2004;166:1269-79.

70. Keightley PD, Davies EK, Peters AD, Shaw RG. Properties of ethylmethane sulfonate-induced mutations affecting life history traits in Caenorhabditis elegans and inferences about bivariate distributions of mutation effects. Genetics. 2000 Sep;156(1):143-54.

71. Estes S, Phillips PC, Denver DR. Fitness recovery and compensatory evolution in natural mutant lines of $C$. elegans. Evolution; international journal of organic evolution. 2011 Aug;65(8):2335-44.

72. Dingley S, Polyak E, Lightfoot R, Ostrovsky J, Rao M, Greco T, et al. Mitochondrial respiratory chain dysfunction variably increases oxidant stress in Caenorhabditis elegans. Mitochondrion. 2010;10:125 - 36.

73. Hicks KA, Howe DK, Leung A, Denver DR, Estes S. In vivo quantification reveals extensive natural variation in mitochondrial form and function in Caenorhabditis briggsae. PloS one. 2012; In Prep.

74. Zielonka J, Kalyanaraman B. Hydroethidine- and MitoSOX-derived red fluorescence is not a reliable indicator of intracellular superoxide formation: another inconvenient truth. Free Radic Biol Med. 2010;48:983-1001.

75. Fujii M, Tanaka N, Miki K, Hossain MN, Endoh M, Ayusawa D. Uncoupling of longevity and paraquat resistance in mutants of the nematode Caenorhabditis elegans. Bioscience, biotechnology, and biochemistry. 2005 Oct;69(10):2015-8.

76. Yanase S, Yasuda K, Ishii N. Adaptive responses to oxidative damage in three mutants of Caenorhabditis elegans (age-1, mev-1 and daf-16) that affect life span. Mechanisms of ageing and development. 2002 Dec;123(12):1579-87.

77. Fujii M, Matsumoto Y, Tanaka N, Miki K, Suzuki T, Ishii N, et al. Mutations in chemosensory cilia cause resistance to paraquat in nematode Caenorhabditis elegans. The Journal of biological chemistry. 2004 May 7;279(19):20277-82.

78. Oeda T, Shimohama S, Kitagawa N, Kohno R, Imura T, Shibasaki H, et al. Oxidative stress causes abnormal accumulation of familial amyotrophic lateral sclerosis-related mutant SOD1 in transgenic Caenorhabditis elegans. Human molecular genetics. 2001 Oct 15;10(19):2013-23. 
79. Gruber J, Tang SY, Halliwell B. Evidence for a trade-off between survival and fitness caused by resveratrol treatment of Caenorhabditis elegans. Ann N Y Acad Sci. 2007; 1100:530-42.

80. Cypser JR, Johnson TE. Multiple stressors in Caenorhabditis elegans induce stress hormesis and extended longevity. The journals of gerontology. Series A, Biological sciences and medical sciences. 2002 Mar;57(3):B109-14.

81. Kell A, Ventura N, Kahn N, Johnson TE. Activation of SKN-1 by novel kinases in Caenorhabditis elegans. Free radical biology \& medicine. 2007 Dec 1;43(11):1560-6.

82. Larsen PL. Aging and resistance to oxidative damage in Caenorhabditis elegans. Proceedings of the National Academy of Sciences of the United States of America. 1993 Oct 1;90(19):8905-9.

83. Aitlhadj L, Stürzenbaum SR. The use of FUdR can cause prolonged longevity in mutant nematodes. Mechanisms Of Ageing And Development. 2010;131(5):3645 .

84. Van Raamsdonk JM, Hekimi S. FUdR causes a twofold increase in the lifespan of the mitochondrial mutant gas-1. Mechanisms Of Ageing And Development. 2011;132(10):519-21.

85. Van Voorhies WA, Curtsinger JW, Rose MR. Do longevity mutants always show trade-offs? Experimental gerontology. 2006 Oct;41(10):1055-8.

86. Durieux J, Wolff S, Dillin A. The cell-non-autonomous nature of electron transport chain-mediated longevity. Cell. 2011 Jan 7;144(1):79-91.

87. Johnson TE, Henderson S, Murakami S, De Castro E, De Castro SH, Cypser J, et al. Longevity genes in the nematode Caenorhabditis elegans also mediate increased resistance to stress and prevent disease. Journal of Inherited Metabolic Disease. 2002;25(3):197-206.

88. Jonassen T, Marbois BN, Faull KF, Clarke CF, Larsen PL. Development and fertility in Caenorhabditis elegans clk-1 mutants depend upon transport of dietary coenzyme Q8 to mitochondria. The Journal of biological chemistry. 2002 Dec 22;277(47):45020-7.

89. Yang W, Li J, Hekimi S. A Measurable increase in oxidative damage due to reduction in superoxide detoxification fails to shorten the life span of long-lived mitochondrial mutants of Caenorhabditis elegans. Genetics. 2007;177(4):206374.

90. Wong A, Boutis P, Hekimi S. Mutations in the $c l k-1$ gene of Caenorhabditis elegans affect developmental and behavioral timing. Genetics. 1995 Mar;139(3):1247-59.

91. Houthoofd K, Braeckman BP, Lenaerts I, Brys K, De Vreese A, Van Eygen S, et al. Axenic growth up-regulates mass-specific metabolic rate, stress resistance, and extends life span in Caenorhabditis elegans. Exp Gerontol. 2002;37(12):1371-8.

92. Altun ZF, Hall DH. Introduction [Internet]. WormAtlas. 2012 [cited 2012 Jun 28]; Available from:

http://www.wormatlas.org/hermaphrodite/introduction/Introframeset.html 
93. Zuo W, Moses ME, West GB, Hou C, Brown JH. A general model for effects of temperature on ectotherm ontogenetic growth and development. Proceedings. Biological sciences / The Royal Society. 2011 Nov 30;279(1734):1840-6.

94. Cutter AD. Sperm-limited fecundity in nematodes: how many sperm are enough? Evolution; international journal of organic evolution. 2004 Mar;58(3):651-5.

95. Hartman P, Childress E, Beyer T. Nematode development is inhibited by methyl viologen and high oxygen concentrations at a rate inversely proportional to life span. J Gerontol A Biol Sci Med Sci. 1995;50(6):B322-6.

96. Goerlich O, Quillardet P, Hofnung M. Induction of the SOS response by hydrogen peroxide in various Escherichia coli mutants with altered protection against oxidative DNA damage. Journal of bacteriology. 1989 Nov;171(11):6141-7.

97. Hodgkin J, Barnes TM. More is Not Better: Brood Size and Population Growth in a Self-Fertilizing Nematode. Proceedings: Biological Sciences. 1991;246(1315):19-24.

98. Ratcliff WC, Hawthorne P, Travisano M, Denison RF. When stress predicts a shrinking gene pool, trading early reproduction for longevity can increase fitness, even with lower fecundity. PloS one. 2009 Jan;4(6):e6055.

99. Yang W, Hekimi S. A mitochondrial superoxide signal triggers increased longevity in Caenorhabditis elegans. PLoS Biol. 2010;8(12):e1000556.

100. Lapointe J, Hekimi S. When a theory of aging ages badly. Cellular and molecular life sciences : CMLS. 2010 Jan;67(1):1-8.

101. Gems D, Doonan R. Antioxidant defense and aging in C. elegans Is the oxidative damage theory of aging wrong? Cell Cycle. 2009;8(11):1681-7.

102. Chen J, Senturk D, Wang JL, Muller HG, Carey JR, Caswell H, et al. A demographic analysis of the fitness cost of extended longevity in Caenorhabditis elegans. J Gerontol Series A. 2007;62:126-35.

103. Lakowski B, Hekimi S. The genetics of caloric restriction in Caenorhabditis elegans. Proceedings of the National Academy of Sciences of the United States of America. 1998 Oct 27;95(22):13091-6.

104. Friedman DB, Johnson TE. A mutation in the age-1 gene in Caenorhabditis elegans lengthens life and reduces hermaphrodite fertility. Genetics. 1988 Jan;118(1):75-86.

105. Corasaniti MT, Strongoli MC, Rotiroti D, Bagetta G, Nisticò G. Paraquat: a useful tool for the in vivo study of mechanisms of neuronal cell death. Pharmacology \& toxicology. $1998 \mathrm{Jul} ; 83(1): 1-7$.

106. Nicotera TM, Block AW, Gibas Z, Sandberg AA. Induction of superoxide dismutase, chromosomal aberrations and sister-chromatid exchanges by paraquat in Chinese hamster fibroblasts. Mutation research. 1985 Sep;151(2):263-8.

107. Kuznetsov AV, Kehrer I, Kozlov AV, Haller M, Redl H, Hermann M, et al. Mitochondrial ROS production under cellular stress: comparison of different detection methods. Analytical and bioanalytical chemistry. 2011 Jun;400(8):238390 . 